\title{
Divertor Currents during type-I ELMs on the DIII-D tokamak
}

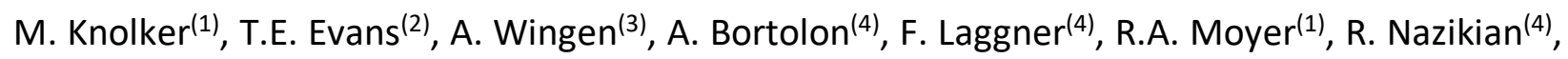 \\ H. Zohm ${ }^{(5)}$
}

(1) University of California at San Diego, San Diego, California

(2) General Atomics, San Diego, California

(3) Oak Ridge National Laboratory

(4) Princeton Plasma Physics Laboratory, Princeton, New Jersey

(5) Max-Planck-Institut für Plasmaphysik, Garching, Germany

\begin{abstract}
Measurements of divertor currents on DIII-D lead to new insights in nonlinear ELM dynamics and a possible mechanism to explain their explosive growth. Rapidly oscillating currents flowing into the divertor before a significant increase in divertor heat flux occurs are measured with an array of shunted tiles and characterized. Extrapolation results in total $n=0$ currents of 5-10 kA flowing into a concentric circle near the strike point. The detected Fourier harmonics appear consistent with a mix of low-n modes $(n<4)$ with currents up to 4 kA. A heuristic framework for ELM currents (ECF) is developed based on thermoelectric origin of the tile currents with flow through regions inside of the nominal separatrix and found consistent with the current measurements. A current flow through the confined plasma leading to increased stochasticity and transport at the plasma edge, could provide a mechanism for additional nonlinear growth as sought for in computational ELM simulations. Results also imply that ELM currents may open the possibility to manipulate the ELM character by perturbations through non-axisymmetric divertor bias or tile insulation.
\end{abstract}




\section{Introduction}

Due to its favorable confinement, the operational regime foreseen for ITER and future fusion power plants is the high confinement mode $(\mathrm{H}$-mode)[1], which comes with periodic relaxations of the edge pressure gradient, termed edge localized modes (ELMs)[2]. As the pulsed heat loads emitted during type-I ELMs will likely cause unacceptable erosion or even melting in the divertor of reactor-scale tokamaks[3], understanding of ELMs is indispensable for fusion reactor operation. In a widely accepted model, the onset of a type-I ELM is explained via the coupling of peeling and ballooning modes[4]. However, during the nonlinear phase-covering the time span after mode onset, the arrival of expelled particles and energy in the divertor prior to the pedestal recovery- is not yet well understood. Simple models predicting heat loads during type-I ELMs still have a threefold scatter compared to experimental data, raising the question of underlying physics mechanisms[5]. Advanced computational ELM simulations point out the need for an additional driver of growth in the nonlinear phase to reproduce experimental results[6-8].

This paper focuses on currents flowing into the divertor tiles during ELMs. Their role in the nonlinear evolution and potential to drive ELM transport is investigated. A heuristic description is developed to explain the origin and impact of the tile currents, termed the ELM current framework (ECF). It is based on a conceptual model predicting thermoelectric currents during ELMs [9]. Additionally, computational efforts confirming the existence of flux tubes as potential carriers of currents between the divertor sides[10] are an important prerequisite for the framework. The ECF quantitatively and self-consistently unites these models with theoretical derivations of thermo-electric tokamak currents based on DIII-D current measurements to explain their contribution to ELM related transport.

The first measurement of currents during ELMs was reported on JET by Harbor et al. in 1989[11] using Langmuir probes. A detailed study on TCV confirmed the findings and was first to mention pre-ELM divertor temperature increases detected by Langmuir probes[12]. Dedicated shunt current resistors connected to isolated tiles facilitated more sophisticated measurements on DIII$D$ and ASDEX Upgrade (AUG)[13], quantifying the size of the ELM currents. Previous studies are concerned with characterizing the divertor currents, for instance regarding the difference between inter-ELM and ELM phase and mainly focused on divertor aspects. The progress introduced in this paper beyond spatial and temporal resolution of the measurements concerns the analysis of the impact of ELM currents.

The structure of the remainder of this paper is as follows: Section 2 gives an overview of the underlying experiments and the tile current array diagnostic on DIII-D and introduces raw measurement results. In Section 3 the tile current evolution during type-I ELMs is described and temporal and spatial variations are analyzed; the section concludes with the determination of mode numbers and rotation patterns of the ELM currents. The magnitude of the currents and their correlation with ELM properties are studied in section 4 . Section 5 is dedicated to the interpretation of the experimental data. A framework is developed to explain origin and impact 
of the tile currents. In Section 6 the results are summarized and discussed within the context of findings on other machines. Conclusions regarding impact and future work are drawn.

\section{Experimental Setup and Measurements}

To achieve generality, this paper is based on multiple DIII-D experiments, different configurations of the tile current array (TCA) and two different divertor constructions, both realized with allgraphite tiles. In the following, the experimental scenarios and TCA configurations are described (an overview is given in table 1).

\section{Current and ELM diagnostics}

The DIII-D tile current array (TCA) has a history of reconfigurations and rededications to address various physics questions $[14,15]$. The most appropriate TCA layout for ELM studies in the lower divertor is shown in Figure 1 with the measurement tiles shown in red. Isolated from their neighbors, each measurement tile is connected via a shunt current resistor ( $\Omega \approx 2.3 \mathrm{mOhm}$ ) to ground. Fast digitizers are delivering sampling frequencies of $200 \mathrm{kHz}$ (and up to $500 \mathrm{kHz}$ in some cases), ideal for ELM measurements, since the duration of a typical ELM-related current flow amounts to $1.5 \mathrm{~ms}$. Regarding the nomenclature, the tile rings are labeled by numbers, with the innermost being 9 and the outermost 14. The tile current monitors are then termed through their machine coordinate and the respective divertor (upper (A) or lower (B)), so that 14B300 refers to the tile current monitor in ring 14 of the bottom divertor at machine coordinate 300 degrees. By design, particularly good coverage is available near the standard strike-point locations. In ring 10, the typical ring of the inner strike point (ISP), 7 out of 48 tiles have fast current monitors and ring 14 , the equivalent for the outer strike point (OSP), 8 out of 72 have fast current monitors. Note that due to data storage constraints, the availability of fast digitized TCA measurements away from the strike points is often limited.

As consequence of the divertor remodeling in 2005, a TCA sensor repositioning and reduction from 40 to 10 (presently active sensors shown in green) took place. To study disruptions the sensors current sensitivity was reduced to measure larger currents resulting in lower signal to noise ratio. Hence, this configuration is not useable for detecting smaller current changes during the ELM onset. However, it is used in this paper to compare the tile current measurements to diagnostic measurements only available after the divertor remodeling (e.g. fast IR data, higher frequency magnetics). The contemporary configuration of the array consists of two circles in the lower divertor in proximity to the OSP (circles 13 and 15 on the elevated shelf structure, contemporary divertor layout not shown in figure 1). Of particular interest are TCA $13 B 068$ and TCA 15B060, as they are in the toroidal vicinity of the 60-degree IR camera on DIII-D. A poloidal cross-section of typical plasma shapes used in this study is shown in figure 2. ISP and OSP reside on the tile current rings 10 and 14 (the other rings of tiles are distributed evenly between them). The separatrix is marked by the thick line, flux surfaces in $\psi_{N}=0.1$ distance are represented by 
dashed (confined plasma) and solid (SOL) lines. Note that this figure does not show the IR camera, since there is no fast IR data available with this vessel/divertor configuration. The interested reader is referred to [16]. The particle exhaust and recycling from the walls are detected by $D-\alpha$ radiation $(656 \mathrm{~nm})$ measurements focused on the outer divertor leg at a 135-degree toroidal position. Typically, the $D-\alpha$ filterscope with the best strike point proximity and signal to noise ratio is selected (shown here is a near X-point channel).

\section{Description of the experiments}

All discharges used for analysis in this paper are in lower single null (LSN) shape. Many of them are chosen from an ELM and Scrape-Off Layer (SOL) study [17], focusing among others on the effect of $B \times \nabla B$ drift direction on ELMs. Representative ELM traces of a discharge with the ion $B \times \nabla B$ drift pointing towards the lower divertor (favorable $\mathrm{H}$-mode power threshold, forward $B_{T}$ on DIII-D) are shown in Figure 3. The plasma current $I_{P}$ is $1.0 \mathrm{MA}$ and the toroidal field is 1.7 T. The ELMs are detected by the D $\alpha$-filterscope (a) with a frequency around $65 \mathrm{~Hz}$. The current measurement of a sensor located near the inner strike point is colored in blue, and the measurement of a sensor near the outer strike point (OSP) is shown in red. Both sensors are connected to tiles positioned at toroidal angle $0^{\circ}$ in machine coordinates. Positive currents indicate a net loss of electrons on the tile, while negative currents indicate net gain of electrons on the tile. During the featured discharge the sampling data amounted to $200 \mathrm{kHz}$. As noticeable in Figure 3 every ELM is correlated with a flow of current through the tiles of comparable amplitude. The maximum values amount to 150-200 A near the inner and -100 A to -150 A near the outer strike point. This would indicate a net flow of negative charge from the inner to the outer strike point. However, there are also spikes in the opposite direction for both rings as shown in the figure (up to -80 A near the inner, $100 \mathrm{~A}$ near the outer strike point respectively). Note that due to constant heating power the plasma conditions are quite stationary: The stored energy is about $0.5 \mathrm{MJ}$ with a stationary $\beta_{N}=1.8(\mathrm{~d})$, and a fairly constant pedestal electron density of $2.3 \cdot 10^{19} \mathrm{~m}^{-3}$ (green). This discharge - as all others in this series- is heated considerably above the LH-threshold $P_{\text {heat }} / P_{L H}=3.0$ with net injected power $P_{\text {heat }}$ and $P_{L H}$ the required power to exceed the $\mathrm{LH}$ threshold[18]. The discharges with the ion $B \times \nabla B$ drift pointing away from the lower divertor are operated at matching plasma conditions (same IP and shape, heating and field strength). A second series of discharges (Table 1) is taken from an ELM squareness study[19] set up with $I_{P}=1.5 \mathrm{MA}$ in forward $B_{T}=1.8 \mathrm{~T}$ and heated by $7 \mathrm{MW}$ of neutral beam power. To compare ELM resolved infrared (IR) camera measurements to tile currents, a recently conducted experiment of type I ELM heat load scaling is analyzed with forward $B_{T}=2.15 \mathrm{~T}$ and $I_{P}=1.5 \mathrm{MA}[16]$. Additionally, an ITER baseline scenario study[20] is included in the analysis. This experiment was also conducted with the contemporary TCA at 1.25 $\mathrm{MA}$ and $1.6 \mathrm{~T}$ (ion $B \times \nabla B$ downwards) with a heating power of $2.5 \mathrm{MW}$. The discharges are heated marginally above the LH threshold and exhibit low ELM frequencies of about $10 \mathrm{~Hz}$. The 
type-I ELM sizes in all discharges considered in this paper range between 15 and $140 \mathrm{~kJ}$

$\left(\frac{W_{E L M}}{W_{M H D}}=2 \%-14 \%\right)$. For simplification the term ELM is used synonymous with type-I ELM in the remainder of this paper; other types are explicitly mentioned.

\section{Current flow and ELM cycle}

The significance and potential role of tile currents are reflected in their magnitude compared to the inter ELM phase currents as well as in their prominent temporal position between rise of magnetic activity in the plasma and the peak of the D $\alpha$ signal as will be shown in the following. Typical ELM evolutions of several large type-I ELMs on DIII-D (>100 kJ, $\approx 10 \%$ ) over the time span of $1.4 \mathrm{~ms}$ are shown in figure 4. This discharge with the present TCA configuration was selected despite reduced spatial accuracy and current sensitivity compared to the old TCA to have fast infrared data for heat flux analysis. The $D$ - $\alpha$-channel is focused on the outer divertor and detects the ELM onset in the SOL (a). Poloidal magnetic probe data at 150 degrees sampling at $250 \mathrm{kHz}$ shows the instability build up in the plasma (b). Regarding the sequence, after the initial growth of the ELM is registered in the magnetics, the interaction of expelled energetic particles with the local plasma leads to an increase of Da radiation and heat flux on the outer divertor (d). Between peak activity in magnetics and filterscope the divertor tile current peaks in the illustrated sensor (c). The temporal shape of the tile current in the outer ring consists of two elements:

- A large spike of up to $400 \mathrm{~A}$ at the time of the first increase in D- $\alpha$-radiation

- A constant current flow with up to $200 \mathrm{~A}$, lasting for about $1.0 \mathrm{~ms}$. This phase often comes in shape of a 'bump' and can mimic the heat flux

The current peak occurs before peak heat flux in the outer divertor measured with the 60-degree IR camera starts to increase (indicated by the dashed line in fig. 3). Note that toroidal TCA location (TCA 13B068) and IR camera position coincide. As the IR camera sampling frequency of $12 \mathrm{kHz}$ is much lower than the sampling frequency of the Da-filterscope or the TCA sensor (here $100 \mathrm{kHz}$ ), it is necessary to carefully analyze measurement time bases. While each data point of the TCA corresponds to the current measurement exactly at that time, the IR data point corresponds to the divertor temperature calculated from the integrated photon count in a $72 \mu \mathrm{s}$ time window at the beginning of each $0.082 \mathrm{~ms}$ sampling interval. Using the THEODOR code[21] the heat flux is then reconstructed for the time step before, as the heat flux causes the temperature increase. This implies that at the current spike (occurring before the dashed line marking the heat flux rise) there is no significant temperature increase in the IR monitored section of the outer divertor. Thus, the ELMs presented in figure 4 are showing that large divertor currents are flowing before the heat flux increases significantly. While it might seem surprising, the consistency of IR and current measurement will be shown in section 5 . 
To resolve the ELM currents, higher sensitivities and sampling rates are required but not provided by the contemporary TCA. Figure 5 illustrates the impact of TCA sampling frequency and toroidal resolution by showing an ELM measured with the old (pre-2005) tile current array. For temporal orientation, the $D \alpha$ radiation and magnetic probe measurements are shown $(a, b)$ to emphasize that tile current oscillations occur during the initial increase of $\mathrm{D} \alpha$ radiation in the divertor both near inner (blue) and outer strike point (red). The respective toroidal location of the sensors is indicated on the top left of each panel. The sampling frequency of the presented TCA is $200 \mathrm{kHz}$, while the $D \alpha$ filterscope frequency is $50 \mathrm{kHz}$. There are three phases distinguishable in this figure:

- the inter-ELM current (prior to $1^{\text {st }}$ dashed line, duration depends on ELM frequency): small currents below $50 \mathrm{~A}$ on all TCA, positive on inner, negative on outer

- the oscillatory phase (between dashed lines, $0.35 \mathrm{~ms}$ duration): peak absolutes of up to $250 \mathrm{~A}$, sign changes

- the main phase (past $2^{\text {nd }}$ dashed lines, about 1 ms duration): currents still large (e.g. 100 A on 10B200), but no sign changes and few or no oscillations

In order to work with the currents during ELMs and understand their impact, the following section will analyze spatio-temporal changes over multiple succeeding ELMs, to describe a typical ELM. 


\section{Tile current flow during an average ELM}

In order to be able to develop a general understanding, tile currents during a typical ELM are characterized and their temporal and spatial variations over multiple ELMs are quantified.

\section{$\underline{\text { Temporal Average }}$}

Under relevant stationary conditions (constant heating, similar pedestal pressure in corresponding inter-ELM phase) the timing sequence of ELM divertor currents for each ELM is similar with the exception of an oscillatory phase at the onset. Figure 6 shows a representative tile current and D- $\alpha$ signal between $2.6 \mathrm{~s}$ and $3.4 \mathrm{~s}$ in discharge 119432, color-coded by time (a). The ELM frequency amounts to $65 \mathrm{~Hz}$ in this segment. As the D- $\alpha$ signal is non-saturated its peak during each ELM is suitable and reliable to be used as reference point for coherent averaging. The resulting average ELM for the D- $\alpha$ signal and the tile current measured at TCA sensor $10 \mathrm{~B} 000$ can be seen in (b) and (c), respectively. The red line indicates the mean of the 48 ELMs. There is broad correlation between succeeding ELMs regarding D- $\alpha$ and TCA signals, except for the time window of 0.35 ms between the two vertical dashed lines. It is a region of strong oscillation, and the oscillations differ from ELM to ELM in amplitude and number. This becomes evident when calculating the standard deviation of the current for each time (d). The peaks of the oscillation are higher on average than the peak during the phase when the heat flux arrives at $\Delta t=0.3 \mathrm{~ms}$, so it is mainly these oscillations that are seen in the overview plot in Figure 3.

\section{Spatial distribution}

Having determined the temporal average of the tile currents during an ELM, it is now interesting to compare the radial and toroidal variation of the current. The drawing of the TCA (Figure 1) illustrates that at a machine angle of 200 degrees there are 5 tiles with current monitors. Radial profiles of current measurements for four tiles at this angle (no fast TCA measurement in ring 13 was available) are shown in Figure 7 for discharges with same shape but different $B \times \nabla B$ direction. The largest currents are measured near the strike points, currents of smaller size are measured in the private flux region.

Reversing the toroidal field direction (keeping Ip direction, so $B \times \nabla B$ drift is now pointing upwards, right column of Figure 7 ) leads to a reversal of tile current direction. While in forward direction the TCA on the inner ring measures positive inter ELM currents and positive deflections during ELMs (119448), the deflections turn negative here in reversed $B_{T}$ (118252), where the outer ring receives positive currents. As the zoom into a single ELM (orange box) shows, the bump is clearly mirrored, whereas ratio of peaks between the oscillatory phase and the bump is smaller on the inner ring in reversed $\mathrm{B}_{\mathrm{T}}$.

As seen from the radial current profiles the vast majority of the ELM currents are flowing through the tile rings near the strike points. Hence, only these will be considered for the toroidal analysis, 
including rotation and mode numbers. Toroidally-resolved current measurements near the strike points during an ELM are shown in Figure 8. The dashed, colored lines in the D- $\alpha$ signal (a) are used to indicate selected measurement times for the tile currents of row 10 near the ISP (b) and row 14 near the OSP (c). In the inter ELM phase (light blue) the measured tile currents show little toroidal variation, with average values of $+40 \mathrm{~A}$ near the inner and $-25 \mathrm{~A}$ near the outer strike point. At the ELM onset there is considerable variation at the toroidal angle of 135 during the oscillatory phase near the OSP (here between yellow and green line). $1 \mathrm{~ms}$ after the D- $\alpha$ peak (purple), the toroidal variation on the ISP has decreased, however, currents have still not returned to pre-ELM values. The standard deviation of TCA currents normalized to their respective means in the oscillatory phase emphasizes a strong $n=1$ deviation near the OSP (d). In the studies of numerous discharges with different edge rotations and ELM frequencies, there has not been a single occasion with an oscillation pattern fundamentally different to the one in Figure 8 (for instance no highly localized occurrence of current spikes). Thus, it can be concluded that the toroidal structure varies relatively slowly (i.e. low-n) and is sufficiently smooth that it can be accurately represented in terms of a low-n Fourier series.

\section{Mode number and rotation}

This section will show that mode numbers inferred from the TCA are within the expected range from peeling-ballooning theory[22] and experimental measurements with fluctuation diagnostics[23]. The toroidal Fourier decomposition $\vec{S}$ of the measured currents $I_{T C A}$ can be found by solving the following matrix equation

$$
\boldsymbol{V}_{\boldsymbol{m o d}} \cdot \vec{S}=\overrightarrow{I_{T C A}}
$$

Here, $\overrightarrow{I_{T C A}}$ represents a vector of the measured tile currents at different toroidal locations within a ring, $\vec{S}$ is the Fourier decomposition of the measured tile currents, and $\boldsymbol{V}_{\boldsymbol{m o d}}$ is the basis matrix of the low-n Fourier reconstruction corresponding to each of the TCA sensors. $\vec{S}$ and $\overrightarrow{I_{T C A}}$ are time-dependent as defined in the appendix. As the total current in the $n=0$ component in a toroidal ring can simply be extrapolated using the sum of all individual TCA measurements $I_{i}$ of that ring and the total number of tiles in the ring $N_{\text {tiles }}$

$$
I_{n=0}=\frac{N_{\text {tiles }}}{N_{T C A}} \cdot \sum_{i=1}^{N_{T C A}} I_{i}
$$

the total current in each phase can be obtained by multiplying the Fourier component with the number of tiles in the respective ring. An example for this analysis of ELM currents near the strike points on discharge 119432 is shown in Figure 9. As there are 7 functional current sensors near 
the ISP (respectively 8 near the OSP) in this discharge mode numbers up to 3 were analyzed. The contribution of these mode numbers can be divided in two groups (a): A mix of the higher mode numbers produces the oscillation in the onset phase of the ELM, whereas the zeroth component is dominant in the post-oscillatory phase. The current in the $n=1$ and $n=2$ component reaches up to $4 \mathrm{kA}$ on the OSP in this discharge, the current in the $\mathrm{n}=3$ phase up to $2 \mathrm{kA}$, indicating that typical helical components are only slightly below the ring current in the oscillatory phase. In the outer ring (lower part of the figure), the Fourier decomposition looks similar with the exception of the sign of the $0^{\text {th }}$ component contribution. While the Fourier decomposition indicates good agreement with a mix of low n-modes (as can be seen by the comparison of black and grey trace, representing experimental current and sum of currents in the Fourier components), note that the possibility to resolve higher mode numbers is restricted by the number of current sensors. If there were high $n$ asymmetries, the TCA would not be able to detect them.

The comparison of total currents near inner and outer strike point in Figure 9 shows that there is a balance. The inter ELM current amounts to $1.7 \mathrm{kA}$ for this forward BT discharge, the matching reverse $B_{T}$ discharge displays a current flow of only $0.9 \mathrm{kA}$ in the opposite direction (not shown here). The resulting ELM peak current values range between 5 and $8 \mathrm{kA}$ (up to $20 \mathrm{kA}$ have been measured on different discharges for large ELMs). This is consistent with previous TCA measurements[24] and magnetic measurements[25]. Unfortunately, a comparison between TCA-measured and magnetics-measured mode numbers is impractical, as the spatial resolution of the old magnetic probe system on these discharges only allows poorly conditioned fits and vice versa (i.e. the present magnetics providing well-conditioned fits can only be compared to poorly conditioned TCA fits). Nevertheless, in agreement with the TCA measurements, a mix of low-n mode numbers is what is typically seen on the magnetics during ELMs in standard H-modes in mid-scale tokamaks[23]. Linear Stability analysis with the ELITE code [4] shows that the most unstable linear modes of these shots are mid-range peeling-ballooning modes around $n=15-20$. Experimental results of a mix low-n modes measured by the TCA in the nonlinear phase are however still in line with the peeling-ballooning model, as nonlinear ELM simulations indicate that the unstable linear modes can beat together and drive a low-n mode in the post-linear growth phase[22,26,27]. Hence, there is correlation between the mode structure in the plasma and in the tile current array. The impact of multiple kiloampere strong currents in the low $n$ modes will be studied in section 5 . 


\section{Correlation between tile currents and ELM properties}

To develop a better understanding for the ELM currents this section will investigate the dependence of the shape of tile currents on plasma or ELM properties further.

\section{Correlation of current size and ELM energy loss}

The inner and outer values of $I_{\text {ring }}$ balance during ELMs within the accuracy of the measurement, as shown in Figure 9. In the inter-ELM period the current is negative near the OSP and positive on the ISP, consistent with an electron flow from the inner to the outer strike point. Consequently, it is now interesting to study two distinct properties of these oscillations - namely amplitude and duration - and their correlation to the plasma energy lost during ELMs measured by magnetic probes. First, the amplitude of the ring current in the oscillatory phase $(6.0 \mathrm{kA}$ for the ELM shown in Figure 9) is compared to the ELM energy loss. Time windows in 20 discharges of the ELM squareness experiment[19] with type-I ELMs were selected based on availability of fast magnetics data and constant strike point position. The ELM energy loss has an offset-linear relationship with the amplitude of $I_{n=0}$ during the oscillatory phase (extrapolated from 5 tiles at the OSP where comparatively more TCA were functional during this series of discharges), as shown in Figure 10. Each data point represents one ELM. The primary uncertainty results from the extrapolation from currents measured in $15 \%$ of the ring circumference. As only large ELMs above $20 \mathrm{~kJ}$ were analyzed due to the measurement accuracy limits, it cannot be stated whether the offset-linear relationship also applies to smaller ELMs. The correlation changes only marginally when considering peak to peak amplitude or the extrapolated sum of the absolute amplitudes of the tile currents.

\section{Oscillation frequency, duration and rotation}

The frequencies of the current oscillations consist of a mixture of high frequency oscillations (>50 $\mathrm{kHz}$ ) and lower frequency oscillations (around $20 \mathrm{kHz}$ ). The frequency is determined as the inverse of the time between two current peaks. Oscillations with higher frequency than $70 \mathrm{kHz}$ were not seen, even in discharges with $500 \mathrm{kHz}$ TCA sampling rate. It is reasonable to compare the oscillatory phase duration and the expected arrival time of ELM expelled ions in the divertor. During an ELM, the ion transit time $\tau_{\|}$from the outer midplane to the divertor can be approximated as the ratio of connection length $L_{C}$ and ion sound speed $c_{S}$ and amounts to[3]

$$
\tau_{\|}=\frac{L_{C}}{c_{S}}=\frac{2 \pi R q_{95}}{\sqrt{\frac{k_{B}\left(T_{I, p e d}+T_{e, p e d}\right)}{m_{I}}}}
$$


where $R$ is the major plasma radius, $q_{95}$ is the safety factor at $\psi_{N}=0.95, m_{I}$ is the deuterium ion mass, $k_{B}$ is the Boltzmann constant and $T_{e, p e d} / T_{I, p e d}$ are the temperatures of electrons and ions at the pedestal top. Typical experimental values on DIII-D yield $\tau_{\|}=0.16-0.22 \mathrm{~ms}\left(q_{95}=\right.$ $\left.4.0, R=2.1 \mathrm{~m}, T_{e, p e d}=0.6-0.9 \mathrm{keV}, T_{I, p e d}=0.7-1.2 \mathrm{keV}\right)$. The duration of the oscillations lasts between $0.05 \mathrm{~ms}$ and $0.3 \mathrm{~ms}$ (determined as maximum time difference between clearly distinguishable peaks). While the order of magnitude is consistent (given that $\tau_{\|}$is an approximative formula and that due to toroidal asymmetry not all tile current dynamics can be captured), the variation of the oscillation duration indicates that there are processes that can lead to a quicker end of the oscillations and there are ELMs in which the oscillations blend into the main phase. Very fast CER measurements have shown that there are two phases during ELMs: fast particle transport out of the pedestal $(<0.3 \mathrm{~ms})$, followed by a slower decrease of temperature (1 ms) [28]. These different transport mechanisms may be related to the dynamics observed with the tile current array.

The analysis of mode rotation and phase patterns emerging from the Fourier decomposition of the oscillatory time window (section 3 ) offers another possibility of comparing plasma to tile current properties. For each of the low $n$ modes $(n=1$ to $n=3)$ a corresponding phase velocity is calculated as average over start and end point of the phase rotation. The emerging current rotation pattern changes strongly from ELM to ELM in whether it rotates at all and which modes rotate. In the few cases with clearly discernable TCA mode frequencies the rotation frequency of the edge plasma measured by CER is clearly exceeded. As the maximum ExB drift frequency in the steep gradient region can be considerably higher than the pure toroidal rotation frequencies (see table 2), a connection to perpendicular drifts will be investigated. The radial electric field $E_{r}$ causes an ExB drift in perpendicular direction of the size

$$
\omega_{E x B}=-\frac{E_{r}}{R \cdot B_{\theta}}
$$

with $R, B_{\theta}$ radius and poloidal magnetic field strength at the outer midplane and the radial electric field computed from force balance for CVI ions as

$$
E_{r}=\frac{\nabla p_{\alpha}}{q_{\alpha} n_{\alpha}}+v_{\phi, \alpha} \cdot B_{\theta}-v_{\theta, \alpha} \cdot B_{\phi}
$$

where $B_{\phi}$ is the toroidal field strength and $\alpha$ refers to the respective species and their charge $q$, pressure gradient $\nabla \mathrm{p}=\frac{d p}{d r}$ and edge density $n$. A comparison of $\omega_{T C A}$ of two different ELMs with rotating $n=1$ and $n=2$ phase, respectively, to $\omega_{E x B}$ profiles obtained from a kinetic EFIT is shown in figure 11 (A kinetic EFIT is an axisymmetric solution to the toroidal equilibrium described by the Grad-Shafranov equation constrained by experimentally measured magnetic probe and flux loop data, and by plasma pressure profile, including a correction for the fast ion pressure and 
inclusion of a Sauter model for the pedestal bootstrap current). $\omega_{T C A}$ rotates in the electron diamagnetic for $n=1$ and in the ion diamagnetic direction for $n=2$. Quantitatively both cases agrees with an origin in the steep gradient region (assuming a frequency bandwidth of $\omega_{E X B} \pm$ $\omega_{\text {dia,el }}$ found in simulations of finite width islands [29]). As the analysis of several discharges conveys (table 2 ), the limits of $\omega_{E X B} \pm \omega_{\text {dia,el }}$ are considerably higher than the pure toroidal rotation frequency at the pedestal top, on the order of the low n mode rotation $\omega_{T C A}$ and at the lower end of the current oscillation frequency. Most of the surveyed ELMs with mode rotation are consistent with a phase rotation in the electron diamagnetic direction. As many ELMs do not show rotation patterns and no clear correlation with drift frequencies can be shown, the rotation analysis indicates several physics elements could be involved. Intense analysis on the resulting magnetic probe measurement of filamentary currents has been done elsewhere, identifying low$\mathrm{n}$ structures as dominant at the ELM onset as likely emerging from the steep gradient region[30].

\section{Comparison to magnetics}

Since currents flowing in plasma and SOL produce their own magnetic fields, it is interesting to compare the tile current array measurements to magnetic probes. Auto spectral density analysis of fluctuation measurements from an internal magnetic probe and TCA is shown in Figure 12. The magnetic probe is localized at 135 degrees toroidal with $1 \mathrm{MHz}$ sampling frequency, while the TCAs are at 115 (ring 10) and 200 degrees (ring 14). While in the inter ELM phases the $n=1$ mode around $20 \mathrm{kHz}$ is clearly detected both on magnetic probe and TCAs (d), the large excitation of the frequency range between 0 and $80 \mathrm{kHz}$ is observed during ELMs, starting with the oscillatory period (b). In the spectrograms $(a, c)$, the ELMs are hence recognizable as the vertical yellow bars. In [30], as a measure for the peaking of the early magnetic perturbations during ELMs, a parameter termed slt for solitariness has been introduced. It is calculated as ratio of the mean value of a Fourier transform of a magnetic signal to its peak. In this case, both magnetics and TCA10 have an slt of 0.041 (outer: 0.049) indicating quantitative correlation of the measurements.

In summary, the correlation between ELM properties and tile currents are found between the tile current amplitude and the ELM size and the power spectrum on magnetic probes with tile current measurements. While rotation is only detectable in the minority of the inspected ELMs, the origin of mode rotation - if present - is likely localized in the outer pedestal and possibly related to diamagnetic or ExB drifts. 


\section{Understanding origin, evolution and impact of the ELM tile currents: A semi-empirical framework}

The previous sections have summarized experimental findings of ELM currents on DIII-D. Due to their large size and critical time of appearance, understanding the origin, nature and impact of these currents is indispensable for a better understanding of ELMs. In the following, an interpretation of the experimental data is made and arranged in the ELM evolution. While it will be shown that the obtained framework is consistent with experimental measurements, it is partly based on hypotheses that by themselves cannot be verified with the available experimental data and are clearly marked as such. As tile currents play a major role in the framework, it is referred to as the ELM current framework (ECF). The ECF describes a mechanism leading to explosive growth and additional transport in the nonlinear ELM phase. The physics of such a mechanism has been identified as missing in contemporary nonlinear ELM simulations by JOREK and M3D $[6,7]$. Without this mechanism ELMs would likely be less violent and of longer duration. While a full nonlinear simulation cannot be presented yet, all stages described in the following can be understood and expected as ad-hoc snap shots during such a simulation. The ECF proposes the following explanation for the tile currents during ELMs:

- after exceeding the peeling ballooning stability threshold, the loss of pedestal energy and particles sets in; the electron heat pulses lead to a rise of plasma temperature in front of the divertor causing thermoelectric currents, marking the beginning of the oscillatory phase (this mechanism has been suggested in a conceptual model for ELMs put forward by Evans[9])

- these currents flow through flux tubes produced by error fields and the ballooning modes between ISP and OSP in the confined plasma. Due to a self-amplifying mechanism, current flow in these flux tubes generates new and larger flux tubes penetrating deeper into the plasma (demonstrated by Wingen [10])

- due to decrease of pedestal pressure and bootstrap current, drive of the flux tube generation mechanism saturates, the drive for the thermoelectric current is reduced and the main phase sets in with the pedestal recovery

Since the thermoelectric currents of the ECF are associated with the oscillatory phase, they do not contribute to initial ELM triggering and they will not alter the onset criteria, which are well described by linear peeling-ballooning theory. The ELM currents are crucial in the nonlinear evolution and the details of the ECF will be explained in the following.

$\underline{\text { Stochastization and existence of flux tubes between divertor strike points through plasma }}$

It has been suggested elsewhere that all of the ELM tile currents are flowing through the SOL[25]. While there are certainly currents in the SOL, among others associated with expelled 
filaments[31], it will be shown that current is flowing through flux tubes in the confined plasma during the oscillatory phase.

To understand the path of current flow, field lines emerging from the divertor tiles are traced employing the MAFOT[32] code. MAFOT is based on the field line tracer TRIP3D[33] and uses the field produced by the plasma current and toroidal field coils as well as error fields or applied fields introduced through various coils (e.g. I- and C-Coils on DIII-D).

MAFOT confirms the conceptually predicted breakup of the separatrix in two parts[9,34-36] - so called manifolds - through the application of non-axisymmetric perturbations. Even small perturbations will lead to separatrix splitting near the X-point, where there is no poloidal magnetic field. The code shows that the two manifolds form an oscillation pattern towards the $\mathrm{X}$-point. At the intersections of the two manifolds within the separatrix, flux tubes are formed connecting the two divertor sides and tiles upon which the strike points reside[10]. At the intersection with the target plates, lobes are formed. These lobes have been verified in simulations among others by the JOREK code[37] and experimentally on DIII-D [38,39] and MAST[40]. The flux tubes are of short length (typically two poloidal turns between the target plates) and inside the stochastic region of the plasma edge, while they themselves are nonstochastic (the field lines within the tube are parallel and of equal length). Larger error fields increase the size and number of these flux tubes. Note that these numerical results are valid for a given moment in time based on the perturbed equilibria in the vacuum field approximation; their temporal evolution cannot be modelled by MAFOT.

The MAFOT code can and has been used to compute additional effects on magnetic topology associated with simulated currents flowing through the short-length connection tubes or the SOL respectively. Previous ELM simulations on DIII-D using the MAFOT code have shown that the IR heat flux footprint geometrically aligns with MAFOT predictions of field line widening at the strike point due to currents flowing through the confined plasma[38]. Agreement between predictions of currents connecting outer and inner divertor through the confined plasma and Langmuir probe measurements has also been found on JET[41]. Note that heat flux patterns on AUG during application of RMPs in L-mode could be modelled without considering error field produced flux tubes in the plasma[42]. Hence, the impact of error field produced flux tubes is likely scenario dependent.

\section{$\underline{\text { Thermoelectric current nature }}$}

The present framework builds on experimental evidence from other devices indicating a temperature increase preceding the bulk particle and heat flux pulse of the ELM. In particular, measurements with Langmuir probes on TCV showed that increased temperature can be detected as early as $0.15 \mathrm{~ms}$ before a heat flux increase[12]. Although comparable measurements were not available for the DIII-D experiments in consideration, these types of observations suggest that thermoelectric effects can be at the origin of the currents observed in DIII-D. After 
the plasma edge exceeds the peeling-ballooning stability threshold, the loss of edge confinement leads to emission of particles and energy towards the divertor. Due to their higher velocity electron conducted heat will arrive within several microseconds at the divertor delivering a heat pulse and leading to local hot spots on both divertor sides[3]. These hot spots in front of each divertor are connected through magnetic field lines to other divertor areas, surrounded by comparatively colder plasma. Consequently, a temperature gradient parallel to B builds up, producing a thermo-electric current with electrons flowing to the hotter divertor side.

For further development of the ECF, theoretical treatment of divertor currents have been revisited, in particular work on thermoelectric currents by Staebler[43]. Staebler's thermoelectric current model shows that due to experimentally confirmed heat load asymmetries in the $\mathrm{H}$-mode between inner and outer divertor $[16,44]$ thermoelectric currents are driven through the plasma between them (whether the magnetic field line is in the confined plasma or in the SOL is not relevant for the Staebler model). The model was found consistent with measurements on DIII-D[45] and AUG[13]. With $T_{I}$ the temperature of the plasma in front of the inner divertor (which is without loss of generality assumed to be colder than $T_{0}$ the temperature at the outer divertor plasma), it predicts a thermoelectric current density of

$$
j_{\text {norm }}=-\gamma \cdot\left[4.04 \cdot\left(\frac{T_{O}}{T_{I}}-1\right)+\ln \left(\frac{1+j_{\text {norm }}}{\left[1-\sqrt{\frac{T_{I}}{T_{O}}} \cdot j_{\text {norm }}\right]^{\frac{T_{O}}{T_{I}}}}\right)\right]
$$

with $j_{\text {norm }}=\frac{j_{\text {par }}}{j_{\text {sat }, I}}$ and $j_{\text {par }}$ the parallel current density along the field line. One can see that in case of no field line temperature gradient $\left(T_{O}=T_{I}\right), j_{\text {norm }}=0$ is the solution to equation (7), while the ion saturation current density $j_{\text {sat, } I}\left(j_{\text {norm }}=-1\right)$ is the upper limit. $\gamma=\frac{\sigma \cdot T_{I}}{j_{\text {sat }, I} \cdot e^{\cdot} \cdot \mathrm{L}}$ is a measure for the quality of the electric conduction between the divertor ends (where $L$ is the length of the field line with conductivity $\sigma$ ).

Strictly speaking, Staebler's thermo-electric current model [43] is developed under the assumptions of negligible pressure gradient between the end points and approximately constant or linearly varying conditions along field lines. In general, these conditions are not expected to be met throughout the later ELM phases. While the exact drive of the current might vary, the sheath conditions determining the maximum current flow will hold during the oscillatory phase. Hence, to check for consistency with the TCA measurements, the absolute size of the current needs to be calculated based on path and connection length between the tiles and the area through which the current flows.

Current flow through confined plasmas and currents flowing in the SOL 
To test the consistency of current flow through confined plasma or SOL with experimental observations, the 80-99 \% ELM phase (as used for stability analysis) in discharge 121560 is analyzed in a $0.5 \mathrm{~s}$ time window around $3 \mathrm{~s}$. The resulting field line connection lengths between the divertor sides computed by MAFOT are presented in figure 13 and show the effect of separatrix splitting by error fields. While the connection length in a) are solely based on a kinetic EFIT and display a well-defined separatrix, the analysis with known error fields from DIII-D C-Coils, F-Coils, and B-coil shows a degenerate separatrix and flux tubes (b). The short connection length flux tubes are recognizable as areas of purple color. At present the plasma response is not included in the framework. While it is well known that $\mathrm{H}$-mode plasmas respond with screening towards external fields, it has been shown elsewhere that including the plasma response reduces the initial penetration depth of flux tube structures but does not fundamentally affect the existence of a large number of flux tubes[46]. Additionally, the contribution of the initial ballooning mode to the flux tube topology is missing. Since rotation of Fourier components is not consistently measured (section 3 ) a competition between error-field-provided, static flux tubes and ballooning evoked rotating flux tubes as seed for the first perturbation is possible. The flux tubes are distorted strongly in the divertor region due to the magnetic field changes, in particular the small poloidal field. By tracing field lines at the boundary of the flux tubes, it can be shown that the tubes are stretched in the toroidal and compressed in the radial direction, so that a radial deposition width in the divertor between 0.001 and $0.02 \mathrm{~m}$ is calculated with a toroidal angle coverage of up to 260 degrees. Additionally, several of the tubes overlay in the same toroidal area. The MAFOT-predicted short flux tubes for discharge 121560 have an average connection length of approximately $90 \mathrm{~m}$. This length, a tile width of $0.12 \mathrm{~m}$ and the MAFOT simulation result of an average radial current inflow width of $0.01 \mathrm{~m}$ can be used to solve equation (7) numerically to obtain the current flowing to a tile.

For SOL currents, field lines outside of the separatrix are traced between the divertor sides. At a certain radius there is no connection to the inner divertor any more, since the field lines intersect with the upper divertor. For major radii between $1.45 \mathrm{~m}$ and $1.49 \mathrm{~m}$ the connection length amounts to $50 \mathrm{~m}$ according to MAFOT, since it is only one poloidal turn (this approximately equals $\left.2 \pi R q_{95}\right)$.

For the numerical evaluation of equation (7) an overview of input parameter range and calculated values for current flow in the SOL and confined plasma is given in table 3 . These ranges are obtained by dividing respective field lines in 1-degree toroidal segments and assigning each its temperature and density according to its grid value from the kinetic EFIT. Non-axisymmetric profile imbalances are hereby neglected, and the assumption is made that the temperature and density in the flux tube equal their local environment. Densities are evaluated from Thomson profiles in the kinetic EFITs to amount to $1.5 \cdot 10^{19} \mathrm{~m}^{-3}$ near the separatrix and approximately $1.0 \cdot 10^{19} \mathrm{~m}^{-3}$ in the far-out SOL. Aside from the connection length taken from MAFOT, the most substantial difference between current flow through SOL or confined plasma results from the 
respective temperatures. They are assumed to be $10-90 \mathrm{eV}$ and $10-400 \mathrm{eV}$ in average along the field line, which is in accordance with contemporary available 2D Thomson divertor spectroscopy on DIII-D [47]. With these assumptions, one obtains a $\gamma$-range of 0.1-1.1 for the SOL path and a range of 0.5-1.1 for the flux tube.

The resulting parallel current $I_{\text {par }}$ for a single tile is shown in figure 14 for different values of the $\gamma$ parameter due to dependence on the temperature ratio $\frac{T_{O}}{T_{I}}$. With a lower $\gamma$ value, a higher temperature difference between the strike points is necessary to drive the same current. The Langmuir probe measurement on the TCV tokamak measuring the initial heat pulse has resulted in a temperature ratio of 2-3:1 [12], so assuming a peak ion target density of $5.0 \cdot 10^{19} \mathrm{~m}^{-3}$ and a DIII-D typical $10 \mathrm{eV}$ on the cold and $30 \mathrm{eV}$ on the hot side [47] corresponds to a peak current of $290 \mathrm{~A}$ for the flux tube path. Average SOL temperatures of $30 \mathrm{eV}(\gamma=0.4)$ are necessary to drive $200 \mathrm{~A}$; for lower temperatures the achievable saturation currents are below the measured values. At approximately $295 \mathrm{~A}$, the predicted ion saturation current is comparable to measured peaks during 121560 . It can be concluded that both current flow through the confined plasma and flow through the near plasma/strike point region with high SOL temperatures are consistent with experimental results (especially in low collisionality, conduction limited SOL cases, with very little temperature gradient).

The strong distortion and interaction of flux tubes near the divertor region could be an explanation for different rotation and mode patterns. Additionally, the consistency of IR heat flux and current measurement during the oscillatory phase presented in section 2 can be shown: Assuming a radial current flow/heat flux width of $0.5 \mathrm{~cm}$ in the divertor and that the electron pulse heats the plasma in front of the tile within a distance of $0.03 \mathrm{~m}$, one yields an affected divertor plasma volume (with the same current deposition width properties as above) of $170 \mathrm{~cm}^{3}$. With an average heat pulse temperature of $50 \mathrm{eV}$ (resulting from a mix of high temperature electrons from the pedestal top and low temperature electrons from the separatrix) near the divertor and an initial local ion density of $10^{19} \mathrm{~m}^{-3}$ a temperature increase from $10 \mathrm{eV}$ to $20 \mathrm{eV}$ is required to reproduce a $50 \mathrm{~A}$ current spike lasting $0.01 \mathrm{~ms}$ (as for instance in TCA 14B045 in figure 4). If the hot electrons from the plasma transfer about $25 \%$ of their kinetic energy (assumed 50 $\mathrm{eV}$ ) to the local plasma (and the rest to the divertor), the electron pulse would need to have 1.4 $10^{14}$ particles which is less than $0.1 \%$ of all electrons in the confined DIII-D plasma so that the heat flux amounts to $0.15 \mathrm{MW} / \mathrm{m}^{2}$ which is slightly below the IR noise level of $0.2 \mathrm{MW} / \mathrm{m}^{2}$ and proves that the initial tile currents can flow without leading to an increased heat flux above noise level.

$\underline{\text { Impact of current flow through confined plasma }}$

Considering the magnetic topology and confinement, a current in the plasma edge (i.e., inside the separatrix) has a much stronger impact on the plasma than a SOL current as shown in Figure 
15. This figure is based on the same equilibrium with error fields as shown in Figure $13 b$ (so the degeneracy of the separatrix has already been removed by the error fields). A typical field line within the near separatrix SOL (a) and within a large flux tube inside the separatrix (c) is traced and color code indicates the temperature along the field line. It is obvious, that the SOL field line is shorter, but also colder in comparison to the flux tube field line. For both cases a current of $500 \mathrm{~A}$ between tiles of ISP and OSP is simulated with MAFOT flowing along the field line and the new magnetic topology represented by divertor connecting field lines is plotted $(b, d)$. Compared with figure 13b, minor changes of field topology occur compared to the original error field plasma when assuming SOL currents (a-b), while in the case of flux tube current in the confined plasma (c-d) the divertor foot is widened and more and larger flux tubes are produced in the plasma as a result of a self-amplifying process, that will be described in the following:

The emerging flux tubes due to the current flow in the initial flux tube penetrate deeper into the plasma (also seen in previous MAFOT runs[32]), i.e. regions of even higher temperature and possibly lower resistance. As the current in the flux tubes increases so will the surrounding stochasticity. In addition, size and number of resonant islands will increase with the current in the flux tubes through the confined plasma so this becomes the instability drive rather than the pressure gradient that was responsible to the initial (linear) instability onset. The expansion of the stochastic region as more heat is fed into the flux tubes, driving more thermoelectric current, is the explosive nonlinear instability that takes over the dynamics following the rather short linear phase. This nonlinearity is quenched when the expansion of the stochastic region (island growth and overlap) slows down and cuts off the heat flux into the flux tubes (note that the number of flux tubes can also increase during this process which enhances the nonlinear growth rate). Thus, it is to be expected that after the onset of the ELM currents (i.e. in the oscillatory period) the majority of current will flow in flux tubes as their resistance will be increasingly lower, while the SOL resistance should remain relatively constant. The process can be compared to a Jacob's ladder with the plasma edge representing the air between the two electrodes (corresponding to the divertor sides). The higher temperature in deeper layers of the pedestal drives the nonlinear growth but finally causes its cessation. The exact mechanism is not understood yet. Nevertheless, a fraction of the currents will also flow in the SOL, as shown in [31], among others driven by filaments or radiation[48].

$\underline{\text { Influence of tile currents on edge stability }}$

After the first measurements of tile currents on AUG, their potential impact on edge stability and $\mathrm{H}$-mode performance was pointed out[13]: From the peeling-ballooning model it is well known that edge currents are a key element of pedestal stability and the currents in the low $n$ Fourier modes can become very large. For instance, in the frame of an instantaneous picture, the current in the $n=3$ component in figure 8 reaches a peak of $3.0 \mathrm{kA}$. This will create a field of $0.02 \mathrm{~T}$ in 3 
$\mathrm{cm}$ distance from the flux tube around the midplane according to Ampere's Law. The $3 \mathrm{~cm}$ are about the distance between the outer SOL and the separatrix. Currents further out are not possible, as the SOL intersects with the vessel. This corresponds to about $1.3 \%$ of the LFS magnetic field and is stronger than all RMP fields on DIII-D, showing the importance of the ELM currents. Going from the instantaneous to the dynamic frame, as long as the flux tubes survive the helical current flowing in them, they can drive RMP fields that contribute to the stochasticity surrounding the flux tubes. As the current in the flux tubes increases so will the surrounding stochasticity, which will affect plasma confinement and could contribute to additional transport.

Validation of the ECF

In order to validate the ECF a balanced double null discharge (DND) with two X-points is analyzed [49]. In a perfectly balanced DND, the inboard and outboard SOL are topologically separated and there is no field line connection between LFS and HFS divertors. Nonlinear ELM simulations of DIII-D have shown that it takes about 40 Alfven times $(0.015-0.03 \mathrm{~ms})$ for the ELM perturbation to spread from the outboard ballooning LFS to the HFS[6]. This delay has been confirmed experimentally in fast reflectometer density measurements on LFS/HFS on AUG[50]. If one assumes that the tile currents are based solely on currents in the SOL caused by perturbations of the plasma through the ELM, one would expect the current change on the high field side to occur delayed compared to the low field side current, since it takes a discrete time window for the perturbation to spread from the LFS to the HFS in the plasma.

Figure 16 shows a reversed $B_{T}$ discharge with a balanced DND around $2.5 \mathrm{~s}$ (119150), and examples of fast TCA signals from lower ISP, OSP and upper OSP (sampled at $200 \mathrm{kHz}$ ). The dashed vertical lines mark a $0.03 \mathrm{~ms}$ time window after the initial current perturbation. One can see the current spikes simultaneously at lower ISP and upper OSP. The experimental result of simultaneous rise of ELM currents measured on HFS and LFS cannot be explained solely based on SOL currents but is indicative of a flux tube passing inside the separatrix between the two sides and as such consistent with the ECF.

In this particular discharge, the plasma shape is changed from an upwards biased DN, to a balanced DN and then to a downwards balanced DN over a time window of 3.0 seconds. To rule out the uncertainty about time point at which the exact DND topology is achieved, in Figure 17, we show for typical ELMs (a selection of 4 ELMs out of 1.5 second time window during the transition was made) how this affects the magnitude of ELM related magnetic perturbation on the HFS and LFS throughout the shape change $(a, b)$. The color code represents the $d R_{\text {sep }}$ parameter, a measure for magnetic balance of the shape defined as radial distance at the outer midplane between flux surfaces connected to the upper and lower X-points. A pure LSN plasma amounts to $\mathrm{dR}_{\text {sep }}$ values below $-0.035 \mathrm{~m}$, a USN plasma has $0.035 \mathrm{~m}$ and above. All signals are shown relative to the peak of the D $\alpha$-signal near the lower OSP. Independent of the bias, the tile currents near inner and outer strike point of the lower divertor rise simultaneously (d,e). As 
expected, for an upwards biased DN case the current increase on the upper divertor LFS (U-LFS) is larger than in the other cases (c) and for LSN-bias the current near the lower OSP resembles the oscillatory - main phase as in purely LSN cases. The current on the L-LFS in the main phase is unusually large and likely due to a current carrying filament (while not shown here, the current on the neighboring tile is considerably smaller in the main phase). These observations are consistent with the ECF interpretation of tile current, since large, simultaneously occurring tile currents are measured on the HFS, even though virtually all power in this balanced DN shape is delivered to the LFS during ELMs [49]. Additionally, while the magnetic SOL perturbation on the HFS is delayed, there is no delay found in ELM related magnetic probe signals on AUG [50] and DIII-D $(a, b)$, consistent with the ECF predicted perturbations induced by current flow inside the nominal separatrix through LFS and HFS.

The connection length plot of the MAFOT simulation of this discharge in the balanced DN time interval (Figure 18a) is similar to the LSN discharges (Figure 13) with the exception that there are two X-points. The figure shows that the error fields of the DIII-D coils have strongest effect near these X-points where a number of lobes is formed (a). Adding a filament with only $150 \mathrm{~A}$ near the lower X-point (in agreement with the early L-OSP measurement) produces a large number of additional lobes on all strike points, in agreement with the measured currents (b). This effect has already been shown in Figure 15d; however (b) demonstrates that flux tube currents near the lower X-point also affect the magnetic topology near the upper X-point. This is because the connecting flux tube (d) is about three times longer than a simple HFS-HFS field line (c) and passes both HFS and LFS. It can be speculated that the ECF addresses the question why the dynamics of single - and double null ELMs are not fundamentally different, even though the topology of the plasma changes considerably: Due to the current in the flux tubes (and here only one filament with $150 \mathrm{~A}$ sufficed to affect divertor footprints) the magnetic perturbation can spread almost instantaneously from the LFS to the HFS independent of the shape.

\section{$\underline{\text { Inter ELM current flow }}$}

While this paper is dedicated to ELM related currents, it is worth mentioning, that the temperature-ratio/current curve shown in Figure 14 is also consistent with inter-ELM measurements and sufficient to explain inter ELM currents. The tile currents amount to $40 \mathrm{~A}$ in forward $\mathrm{B}_{\mathrm{T}}$ scenarios, which corresponds to a realistic temperature ratio of $1.2: 1$. In forward $\mathrm{B}_{T}$ the outer divertor receives more heat load than the inner divertor on DIII-D and heats up stronger, leading to a net inflow of electrons due to a thermo-current in agreement with the theory[43]. Consistent with the reversal of the divertor heat load asymmetry with $\mathrm{B}_{T}$ direction[44], the tile currents change direction in reversed $\mathrm{B}_{\mathrm{T}}$ and are smaller in amount (only 10-20 A are measured). The exact conductivity along the flux tubes is between its two extreme limits: 
- low temperature divertor flux tube with $\gamma=0.1$ : as the flux tubes are connected to the divertor/SOL region with much lower temperatures, the low-conductivity/high resistance limit is formed by assuming SOL conditions in the flux tubes, due to the high parallel transport along them. This is unrealistic in so far as the low temperature flux tube in the hot confined plasma would lead to very high perpendicular transport

- high temperature core flux tube with $\gamma=1$.1: the high-conductivity/low resistance limit is formed by assuming temperatures in the flux tube corresponding to their nested flux surface. This is unrealistic as one would assume a steep gradient in temperature at the transition from confined to SOL plasma

Although these assumptions for the inter-ELM phase both SOL current flow and current flow through flux tubes are consistent with the measurements, further quantitative modeling is necessary to understand the effect of temperature profiles on conductivity and the plasma response. 


\section{Summary and outlook}

Unfavorable extrapolations of type I-ELM heat loads towards ITER emphasize the widespread need of a more thorough understanding of dynamics in the nonlinear ELM phase. For this purpose, the role of currents flowing into the divertor as potential driver of explosive growth during the instability was elucidated in this paper. These ELM currents have been previously observed on mid-size and smaller tokamaks. Here, important new experimental findings will be summarized:

- At the ELM onset, there are divertor currents flowing into and out of tiles near the strike points, before the heat flux increase is measured by IR thermography. Currents of inner and outer strike points display opposite sign, reversing with a change of $\mathrm{B}_{\mathrm{T}}$ direction.

- The current flow during ELMs consists of an initial oscillatory phase (<0.3 ms) with high frequency oscillations $(5-67 \mathrm{kHz}$ ) and a subsequent bulk phase, characterized by large but steady currents

- Current flow is radially strongly localized near the strike points, however small currents are measured in the private flux region during ELMs

- Current peaks between 0.3-0.5 kA per tile are recorded, which amounts to 5 - $20 \mathrm{kA}$ current in the ring. Sign and size of the currents agree with a thermoelectric nature and origin of the currents with the ion saturation current on the colder side as the upper limit

- While no consistent rotation pattern is found, Fourier analysis indicates a mix of low $\mathrm{n}$ modes $(<3)$ within the measurement resolution in agreement with nonlinear peelingballooning theory

- Correlation between magnetic probes and TCA measurements exists for core modes in the inter-ELM phase and in form of wide frequency response during ELMs

- There is a weak correlation between peak current amplitude in the oscillatory phase near the strike points and ELM size

Thermo-currents during the inter-ELM and ELM phase of comparable size to DIII-D were also measured on AUG with a tile current array located at three toroidal positions [13,51]. The reversal of current flow with change of the $B \times \nabla B$ direction was pointed out on AUG [52]. Additionally, the same paper reports a proportionality between asymmetry of ELM energy deposition between inner and outer divertor and the exchanged charge (integral over the current flows between divertor legs, with both measured against ground). For reversed $\mathrm{B} \times \nabla \mathrm{B}$ drift direction the proportionality constant is twice as high, so double the charge is exchanged between divertor sides for a similar ELM size. A slight decrease in current amplitude was observed with $\mathrm{B} \times \nabla \mathrm{B}$ drift reversal in this work too, however the inter ELM amplitude has decreased as well: Hence, this effect might be due to different plasma temperature conditions in 
front of the divertor consistent with reduced heat flux measurements in matching reversed $B_{T}$ cases.

Based on these experimental findings and previous work a new framework was developed elucidating the origin, role and effect of currents during ELMs. Potentially addressing mechanisms for additional nonlinear growth as sought for in large scale simulations the framework describes the drive of explosive growth provided by currents and is hence named the ELM current framework (ECF). While the measurements agree well with the predictions of the framework it is at present still based on ad-hoc simulations. The successive stages of the ECF and the experimental findings or simulation results, which the stages are based upon are outlined in the following

- after exceeding the peeling-ballooning stability threshold, loss of energy and particles sets in at the pedestal; the electron heat pulses lead to a rise of plasma temperature in front of the divertor as measured by Langmuir probes on TCV

- thermoelectric currents flow from the hot divertor plasma to colder parts on the other divertor side through flux tubes in the confined plasma. The current size agrees well with calculations based on flux tube areas on the tiles predicted by the MAFOT code and a model for thermoelectric current flow along field lines developed by Staebler with the ion saturation current as upper limit.

- these flux tubes are produced by error fields and the ballooning mode at the plasma edge in a competing process causing different rotation patterns in the tile current array. In a self-amplifying mechanism, more and larger flux tubes are produced due to thermoelectric currents in the tubes. The growth in number and size of the flux tubes can be confirmed by MAFOT simulations and outcomes have been verified with comparisons of IR footprints. The current flow through the plasma edge also explains the high correlation between power spectral densities of TCA and magnetics.

- The increasing stochastization caused by current flow in the flux tubes facilitates access to deeper layers of the pedestal causing additional heat and particle transport

- when the pedestal has regained stability due to a nonlinear saturation mechanism and the arrival of ions in the divertor has balanced thermal gradients, the drive for the thermoelectric current ceases and the transition to the filamentary phase sets in with the pedestal recovery

While the tile current size is also consistent with SOL currents in vicinity of the separatrix, ELM current measurements in balanced DN scenarios cannot be explained by pure SOL currents: Simulation results of finite time ( $\sim 0$ Alfvén times) for the magnetic ELM perturbation to spread from LFS (where the outboard ballooning sets in first) to HFS disagree with the measured 
simultaneous onset of HFS and LFS currents. The ECF provides a mechanism for simultaneous current onset through flow in confined plasma flux tubes.

\section{Outlook}

While the ELM current framework presented in this paper is consistent with experimental data it is based on instantaneous field line tracing and current calculations. Remaining questions comprise of the influence of error fields on potential locking and the trigger mechanism: Can one detect changes in the ELM behavior by varying the size of the error fields and induce locking? Does the growth of the peeling ballooning mode itself contribute to the generation of currentcarrying flux tubes? What role do the plasma response and the filaments play in the cycle? Can the increase of stochasticity break up flux tubes? What is the exact saturation mechanism? In order to further understand the impact of the tile currents on transport and evolution of type I ELMs it is important to implement tile currents as boundary condition into non-linear simulations such as JOREK. Quantitative modeling will also lead to a better understanding of how the flux tube resistance in deeper layers of the pedestal behaves given the strong dependence of conductivity on its temperature $\left(\sigma \sim T_{e}^{1.5}\right)$. This way, the hypotheses of the ECF could be tested and in a second step, the effects of manipulating tile currents could be estimated. This is a major physics question unfolding into two directions:

- If using insulated tiles (that is not only against their neighbors but also against the torus and maybe only temporarily), can the characteristics of ELMs (e.g. currents in oscillatory phase) be affected and ultimately ELM mitigation be achieved, as a potential stochasticity-enhancing mechanism is suppressed?

- Can tile biasing be used to drive significant helical current through the edge of the confined plasma to impact the ELM crash or general ELM behavior?

Theoretical considerations indicate the possibility to drive currents near the plasma edge in order to suppress ELMs[53]. Most of the previous bias experiments on mid-size tokamaks used axisymmetric setups in the form of a ring or symmetrically distributed probes[54-56]. Given the results of current size and non-asymmetric distribution in this paper, it is understood that the results seen were local and had no global effect on ELMs. An experiment to test manipulation of tile currents would have to be based on a non-axisymmetric setup to drive helical currents. The differences between DC biasing and feedback on magnetic activity on ELM crashes would have to be explored experimentally. In contrast to RMP ELM suppression, QH-mode plasmas or ELM pacing that all come with operational constraints, using tile insulation or biasing - if successfully demonstrated - might be a path to mitigation of ELM heat loads on the divertor independent of plasma conditions. 


\section{Appendix}

\section{Mode number derivation}

In equation (1) $V_{\text {mod }}$ takes the form of

$$
V_{\text {mod }}=\left[\begin{array}{cccccc}
1 & \sin A & \cos A & \ldots & \sin n A & \cos n A \\
1 & \sin B & \cos B & \ldots & \sin n B & \cos n B \\
\ldots & \ldots & \ldots & \ldots & \ldots & \ldots \\
1 & \sin E & \cos E & \ldots & \sin n E & \cos n E
\end{array}\right]
$$

Whereby $A, \ldots . . E$ represent the angles in machine coordinates of the different TCA and $n$ is the highest desired mode number for analysis. The $V_{\text {mod }}$ dimensions are number of TCA $x$ (2xmaximum mode number+1). The tile current array is consequently represented as

$$
\overrightarrow{I_{T C A}}=\left[I_{A} I_{B} \ldots \ldots I_{E} \quad\right]
$$

The solution $\vec{S}$ of (1)

$$
\vec{S}=V_{\bmod }{ }^{-1} \cdot \overrightarrow{I_{T C A}}
$$

is then found using a matrix solver. Its length is $j=2 n_{\max }+1$ with $n_{\max }$ the highest resolved mode number and it is split in the various mode contributions.

$$
\overrightarrow{S_{\text {reconstr }}}=\left[\begin{array}{c}
1 \\
S[1]+i S[2] \\
\cdots \\
S[j-2]+i S[j-1]
\end{array}\right]
$$

out of which then amplitude and phase of the various modes can be extracted by known methods. The change of the phase over time indicates the rotation of the mode. 
TABLES

\begin{tabular}{|c|c|c|c|c|c|}
\hline Series & $\begin{array}{l}\text { Analyzed discharge } \\
\text { range }\end{array}$ & TCA & $\mathrm{B}_{\mathrm{T}}[\mathrm{T}]$ & $\mathrm{I}_{\mathrm{P}}[\mathrm{MA}]$ & $f_{E L M}[H z]$ \\
\hline ELM BT characterization & $\begin{array}{c}118210 \\
118250-118255 \\
119432-119450\end{array}$ & Old & $\begin{array}{l}+2.15 \\
+1.7 \\
-1.7\end{array}$ & 1.0 & $20-70$ \\
\hline ELM squareness & $121553-121571$ & Old & -1.8 & 1.5 & $38-80$ \\
\hline RMP ELM suppression & 123301 & Old & -2.0 & 1.5 & 0 \\
\hline Long Pulse ITER baseline & 147140 & New & -1.6 & 1.25 & $5-20$ \\
\hline ELM heat load & 169509 & New & -2.15 & 1.5 & $10-25$ \\
\hline
\end{tabular}

Table 1: Overview of discharges analyzed or referred to in this paper. All shapes are LSN, negative $\mathrm{B}_{\mathrm{T}}$ (forward $\mathrm{B}_{\mathrm{T}}$ ) indicates ion $B \times \nabla B$ pointing to the lower divertor.

\begin{tabular}{|c|c|c|c|c|c|c|c|}
\hline Discharge & $\begin{array}{c}\mathrm{E}_{\mathrm{R}} \\
{[\mathrm{kV} / \mathrm{m}]}\end{array}$ & $\begin{array}{c}\omega_{\text {dia, }} \\
{[\mathrm{kRad} / \mathrm{s}]}\end{array}$ & $\begin{array}{c}\omega_{\text {dia, },} \\
{[\mathrm{kRad} / \mathrm{s}]}\end{array}$ & $\begin{array}{c}\omega_{\text {ExB }} \\
{[\mathrm{kRad} / \mathrm{s}]}\end{array}$ & $\begin{array}{c}\omega_{\text {tor,ped }} \\
{[\mathrm{kRad} / \mathrm{s}]}\end{array}$ & $\begin{array}{c}\omega_{\text {TCA }} \\
{[\mathrm{kRad} / \mathrm{s}]}\end{array}$ & $\begin{array}{c}\omega_{\text {osc }} \\
{[\mathrm{kRad} / \mathrm{s}]}\end{array}$ \\
\hline 119432 & -30 & -21 & 142 & -44 & -15 & $6-84$ & $35-250$ \\
\hline 119433 & -26 & -18 & 130 & -38 & -8.8 & $-75-53$ & $23-310$ \\
\hline 119434 & -22 & -24 & 188 & -32 & -9.2 & $-28-82$ & $48-250$ \\
\hline 119440 & -12 & -22 & 72 & -18 & -12 & $22-69$ & $29-310$ \\
\hline 121560 & -43 & -38 & 115 & -46 & 0.4 & - & $78-157$ \\
\hline 118252 & -15 & -14 & 54 & -21 & -8.8 & - & $35-250$ \\
\hline
\end{tabular}

Table 2: Overview of rotation quantities for three different discharges around $3.0 \mathrm{~s}$ : Toroidal pedestal Carbon rotation frequency $\omega_{\text {tor,ped }}$, radial electric field $E_{R}$ and diamagnetic contributions $\omega_{\mathrm{dia}, C}$ and $\omega_{\mathrm{dia}, e}$, ExB rotation frequency $\omega_{\mathrm{ExB}}$, TCA phase rotation $\omega_{\text {TCA }}$ and current oscillation frequency $\omega_{\text {osc }}$. 


\begin{tabular}{|c|c|c|}
\hline & $\begin{array}{c}\text { ELM current through flux tubes } \\
\text { in confined plasma }\end{array}$ & ELM current in SOL \\
\hline$n_{e}\left[10^{19} \mathrm{~m}^{-3}\right]$ & $1.0-3.0$ & $1.0-1.4$ \\
\hline$n_{i}\left[10^{19} \mathrm{~m}^{-3}\right]$ & $1.0-3.0$ & $1.0-1.4$ \\
\hline$T_{e}[\mathrm{eV}]$ & $10-400$ & $10-90$ \\
\hline$L_{C}[\mathrm{~m}]$ & $90 \pm 5$ & $50 \pm 5$ \\
\hline$n_{I}\left[10^{19} \mathrm{~m}^{-3}\right]$ & $0.53-1.15$ & $0.07-1.07$ \\
\hline$\tau_{i e}[\mu \mathrm{s}]$ & $5.3-11.4$ & $0.3-5.3$ \\
\hline$\sigma\left[10^{24} \mathrm{Sm} m^{-1}\right]$ & $0.53-1.14$ & $0.07-0.95$ \\
\hline$\gamma$ & $210-260$ & $50-260$ \\
\hline$T_{O} / T_{I}[\mathrm{eV}]$ & $30 \mathrm{eV} / 10 \mathrm{eV}$ & \\
\hline $\mathrm{I}\left(\frac{T_{O}}{T_{I}}=3.0\right)[\mathrm{A}]$ & & \\
\hline
\end{tabular}

Table 3: Comparison of plasma parameters and resulting divertor currents in A for flow through flux tubes in confined plasma or through SOL. Input parameter for equation (9) and resulting tile current at a divertor temperature ratio of 2:1. 


\section{FIGURES}

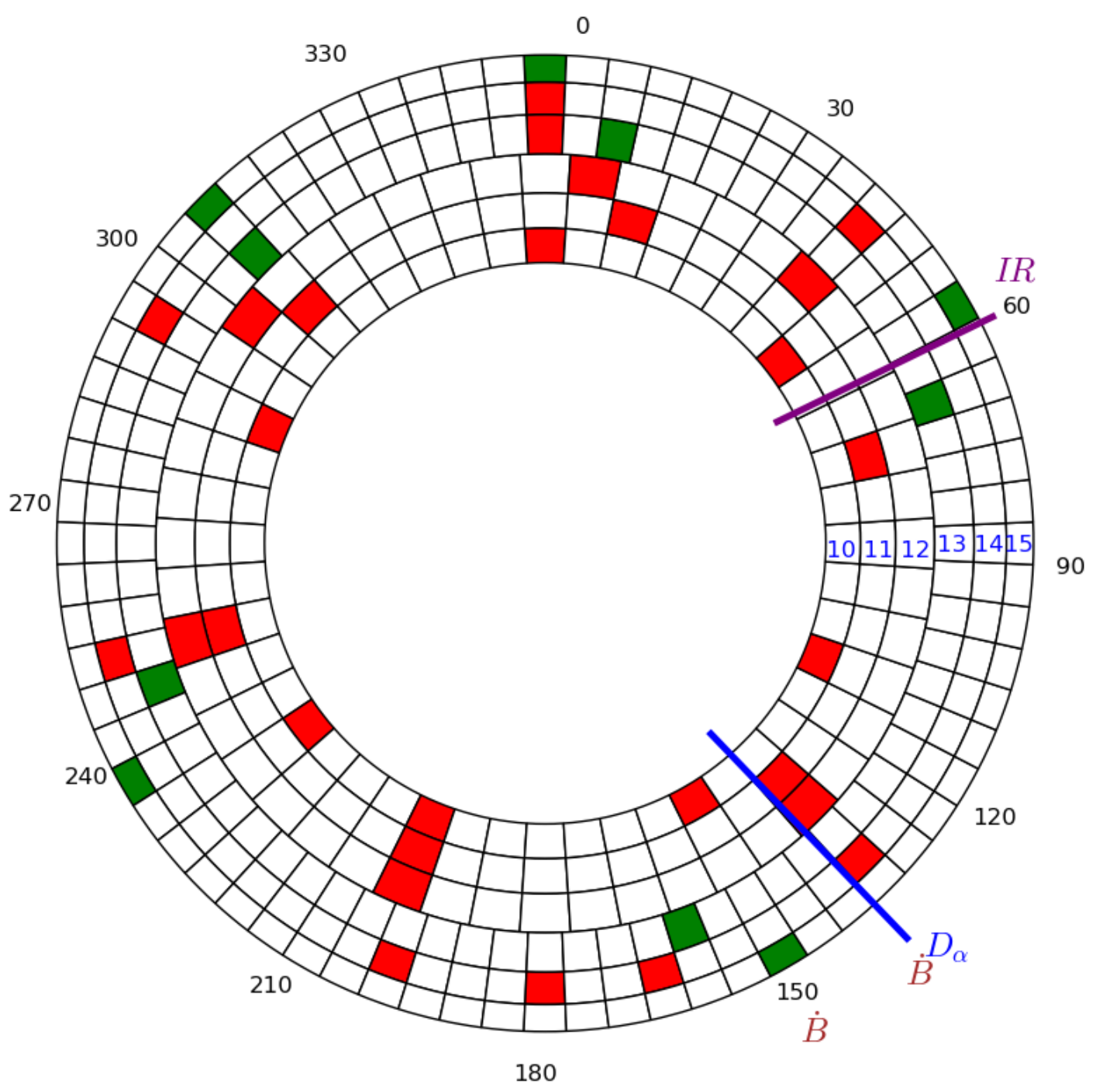

Figure 1 Lower divertor from 2002-2005 with toroidal machine coordinates and tile current sensors in red, present day measurement positions indicated in green: The nomenclature of the tile rings from 10-15 is indicated in blue, as well as projected sight lines of IR (purple), Da (blue) and positions of fast magnetic probes (brown). 


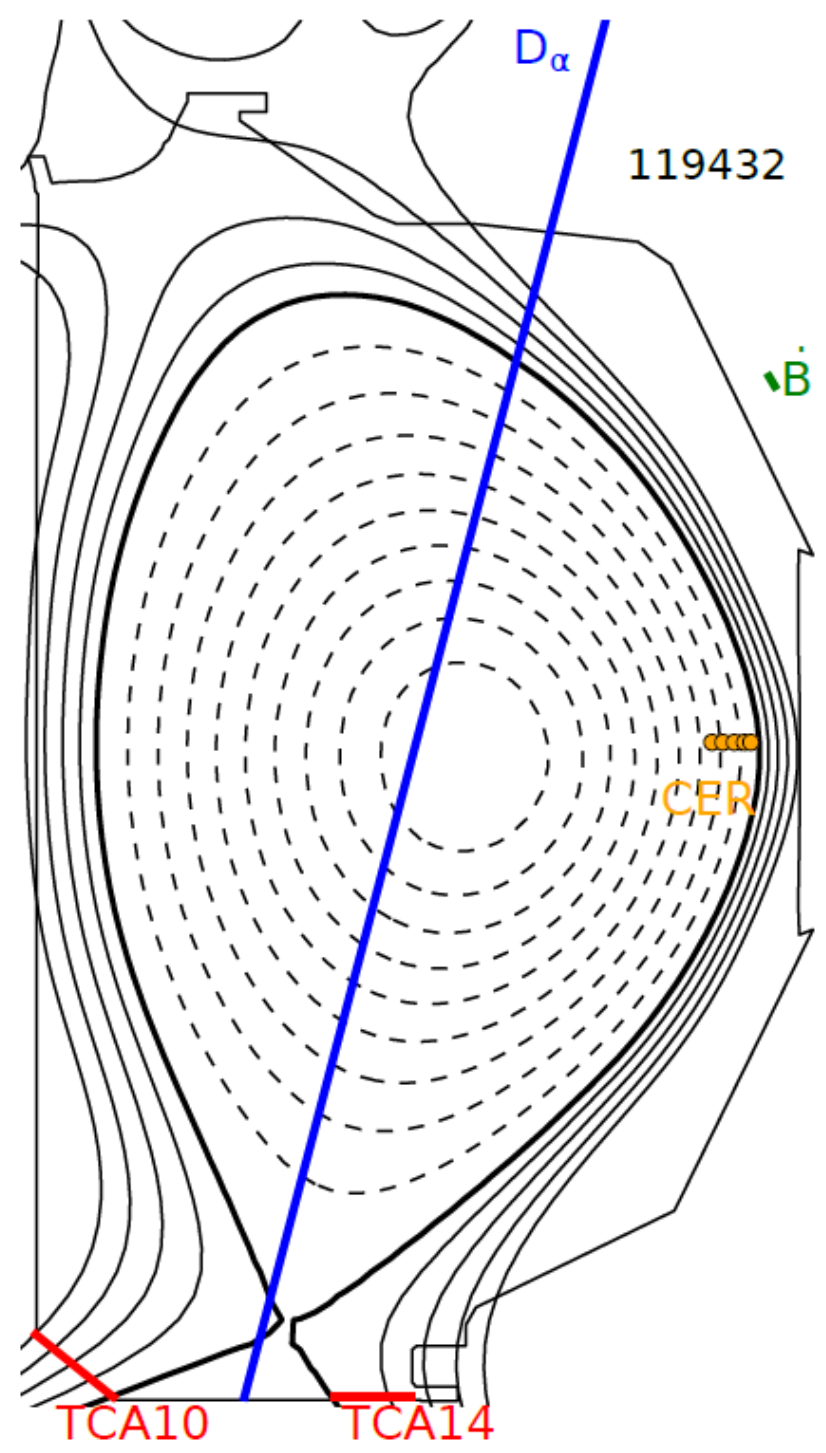

Figure 2 Poloidal cross-section of typical lower single Null diverted DIII-D plasma in the old divertor configuration. Tile current array channels near inner and outer divertor (red), edge CER channels (yellow), fast magnetic probes (green) and representative D $\alpha$ channel near X-point (blue) 


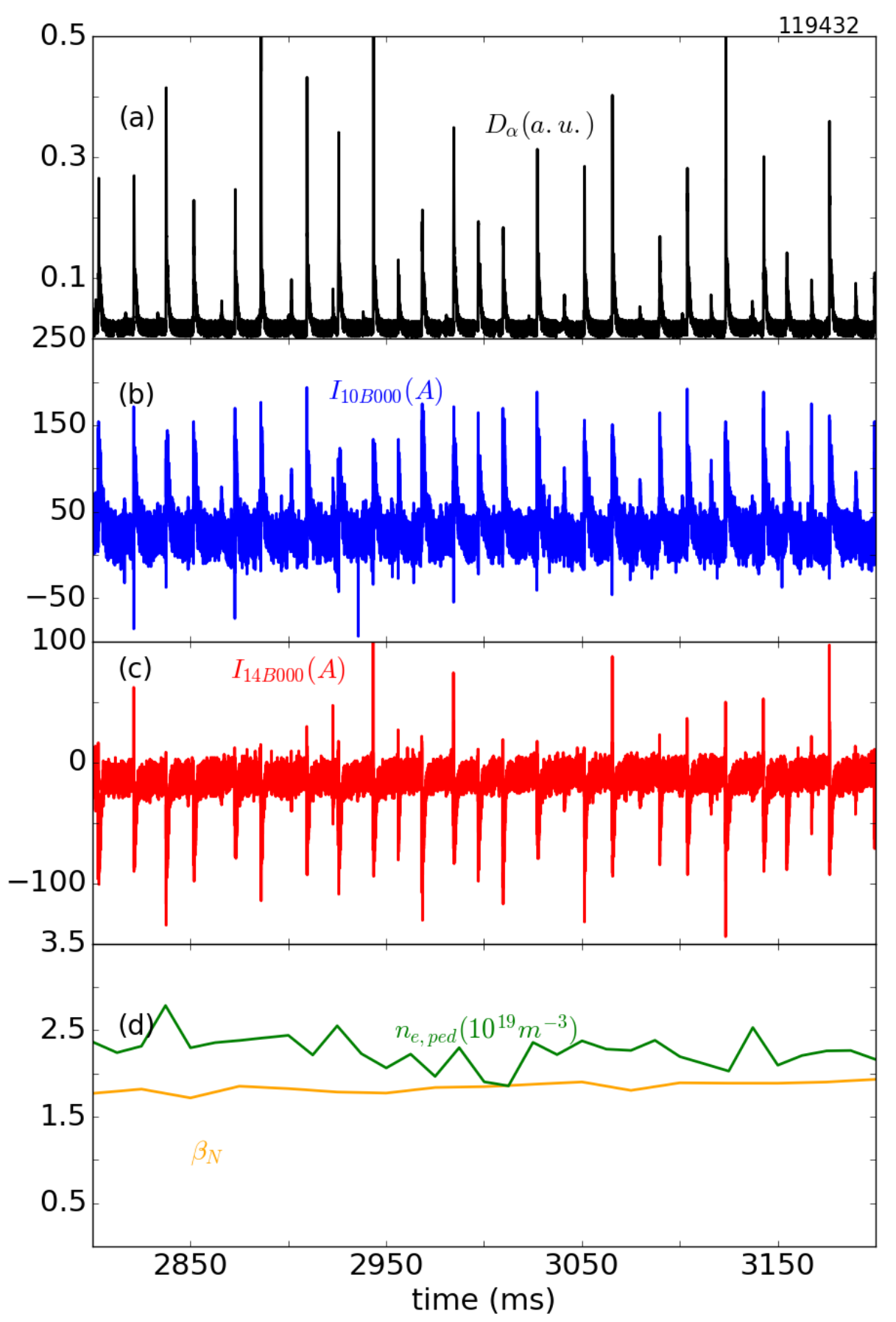

Figure 3 Discharge evolution of 119432 between 2.8 and $3.2 \mathrm{~s}$ : a) Da emission, b) current measurement on single tile near ISP c) and near OSP d) pedestal density (green) and normalized beta (yellow) 


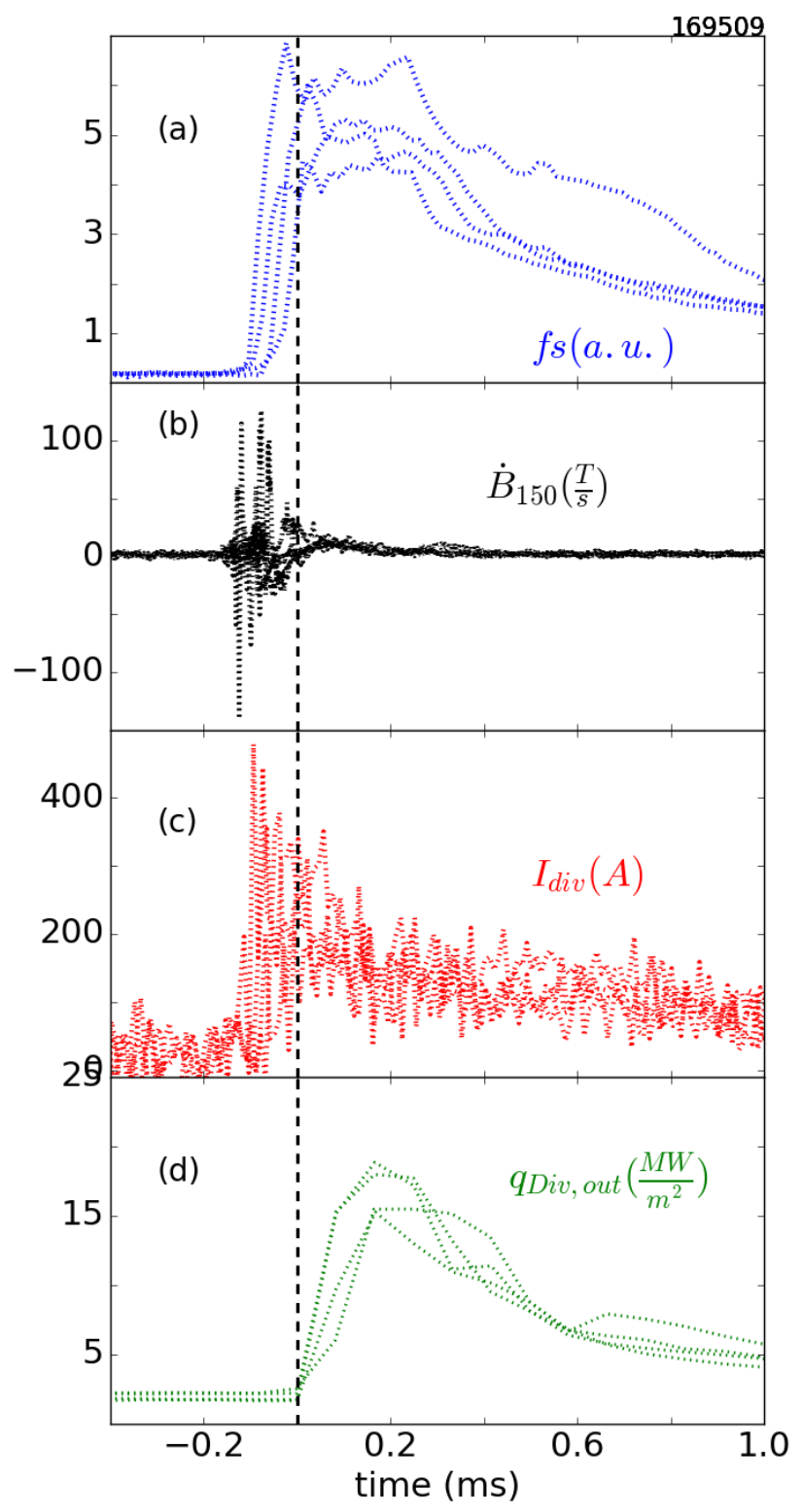

Figure 4 Current Precursor for successive type-I ELMs in divertor on discharge 169509, $1.4 \mathrm{~ms}$ time window: a) D- $\alpha$-filterscope at 135 degree toroidal (50 kHz sampling rate), view on outer divertor b) poloidal magnetic fluctuation measurement at 150 degree toroidal $(250 \mathrm{kHz}$ sampling rate) c) Divertor Current measured at 68 degree in row 13 (100 kHz sampling rate) , d) peak outer heat flux in $\mathrm{MW} / \mathrm{m}^{2}$, IR camera at 65 degree (12 kHz sampling rate) 

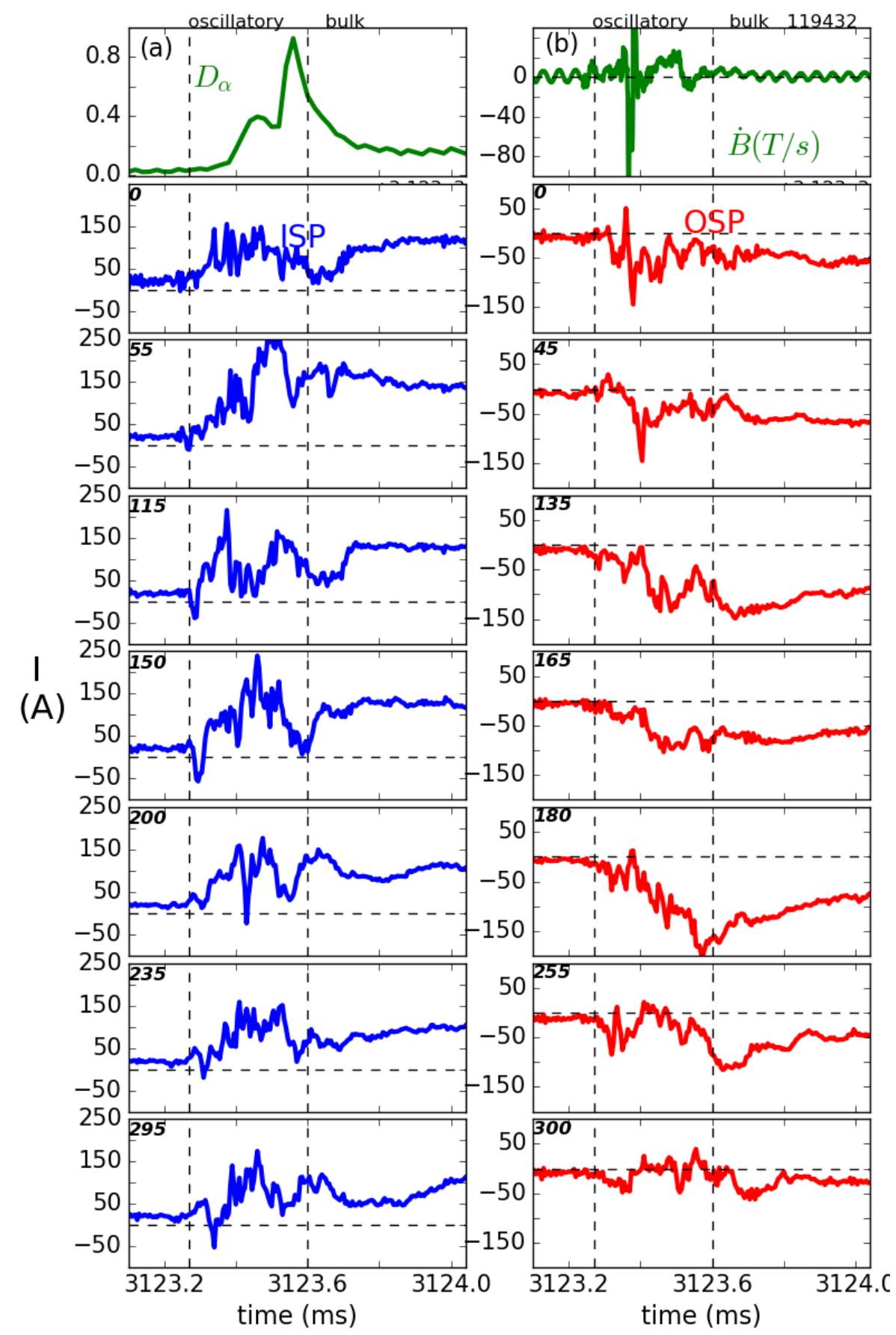

Figure 5 Tile currents during an ELM on discharge 119432 and D $\alpha$ radiation (a) and magnetics (b) for temporal orientation. Remaining panels show oscillations of tile current during the increase in Da radiation are measured near inner (blue, TCA ring 10) and outer (red, TCA ring 14) strike point. The toroidal position is indicated by the bold number on the top left. Overall $0.8 \mathrm{~ms}$ are shown, covering pre-ELM and initial ELM phase. 
119432
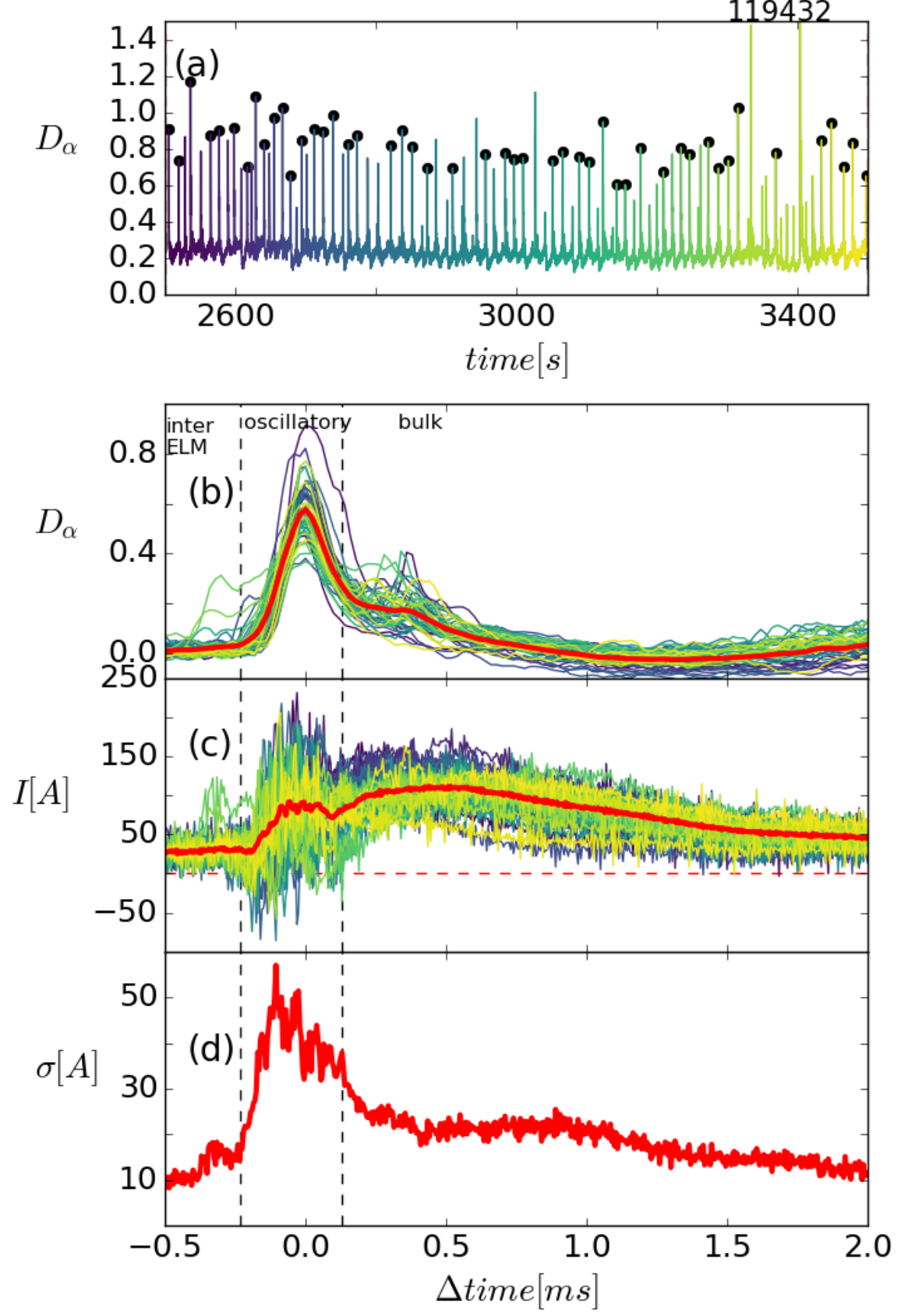

Figure 6 Temporal average of ELMs on 119432 between 2.5 and $3.5 \mathrm{~s}$ : a) D $\alpha$ trace b) Da relative to its peak of individual ELMs with the average in red c) tile current in TCM $10 \mathrm{~B} 000$ relative to Da peak with the red line marking the average d) standard deviation of the tile current 


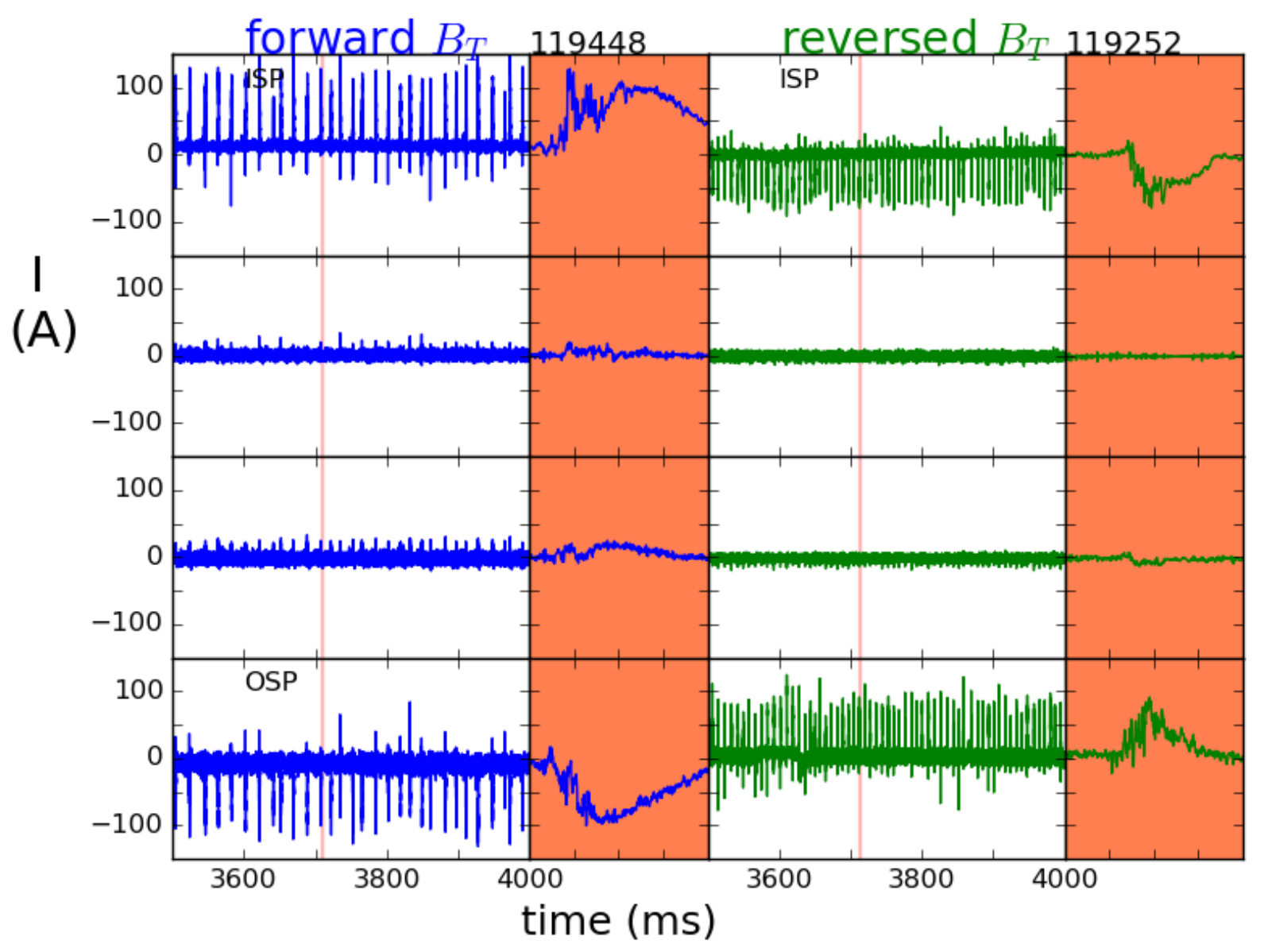

Figure 7 Comparison of TCA signals during forward Bt (blue, 119448) and reversed Bt (green 118252) between $3.5 \mathrm{~s}$ and $4.0 \mathrm{~s}$ at 200 degrees in machine coordinate from inner 10 ring (a) to outer 14 (d) ring. The used signals here are from the rings 10,11,12 and 14 (from top to bottom). The orange regions are a zoom into a single ELM from $3711.5 \mathrm{~ms}$ to $3713.5 \mathrm{~ms}$ to resolve the oscillations. 


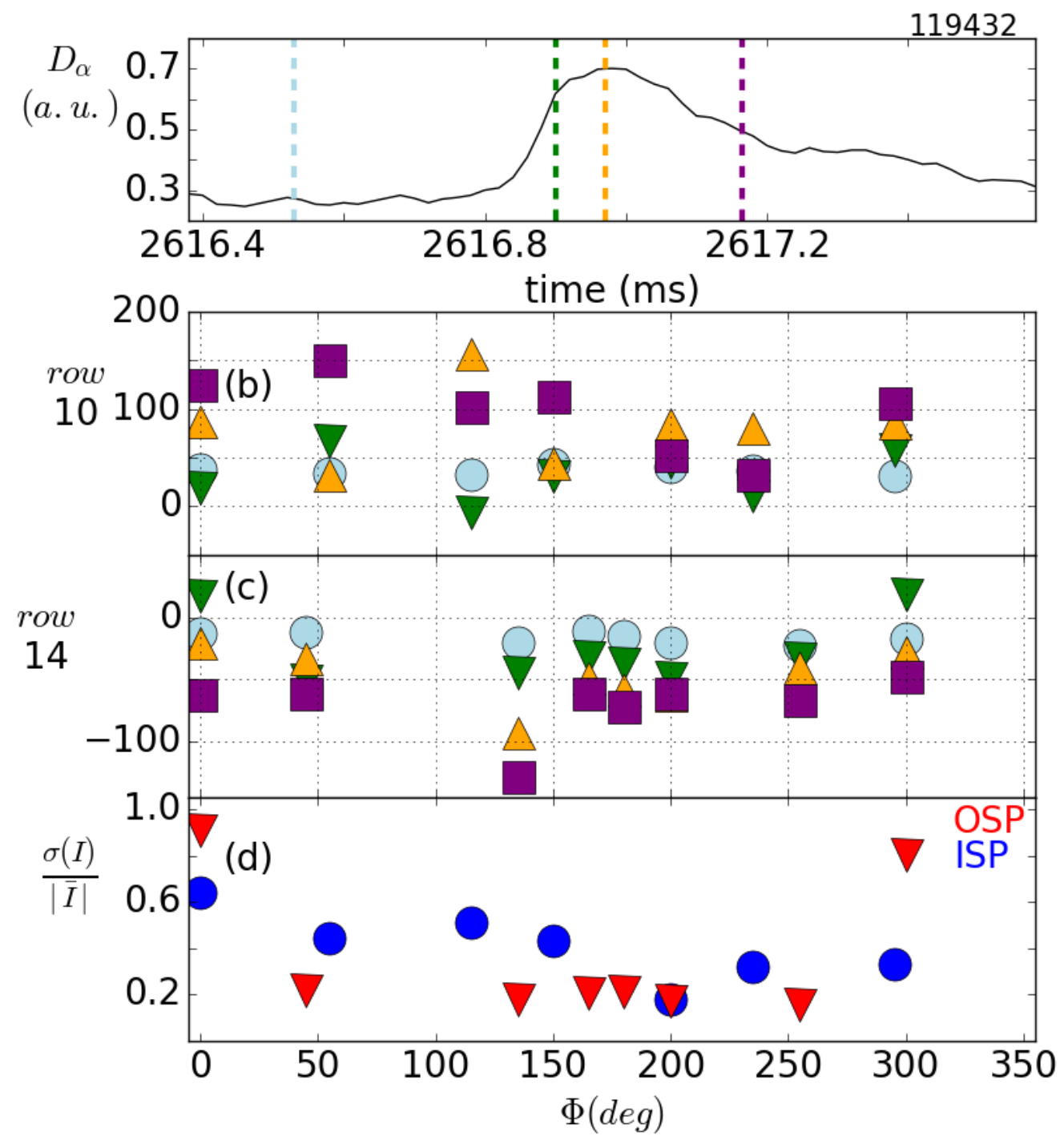

Figure 8 Toroidal current measurement of a single ELM in 119432: a) D $\alpha$ signal with color-code to identify position and succession for $b$ ) tile current as monitored near the inner strike point and c) outer strike point d) ratio of standard deviation and absolute mean of tile currents near ISP and OSP during oscillatory phase between 2616.90 (green line) and 2616.98 (yellow line). 


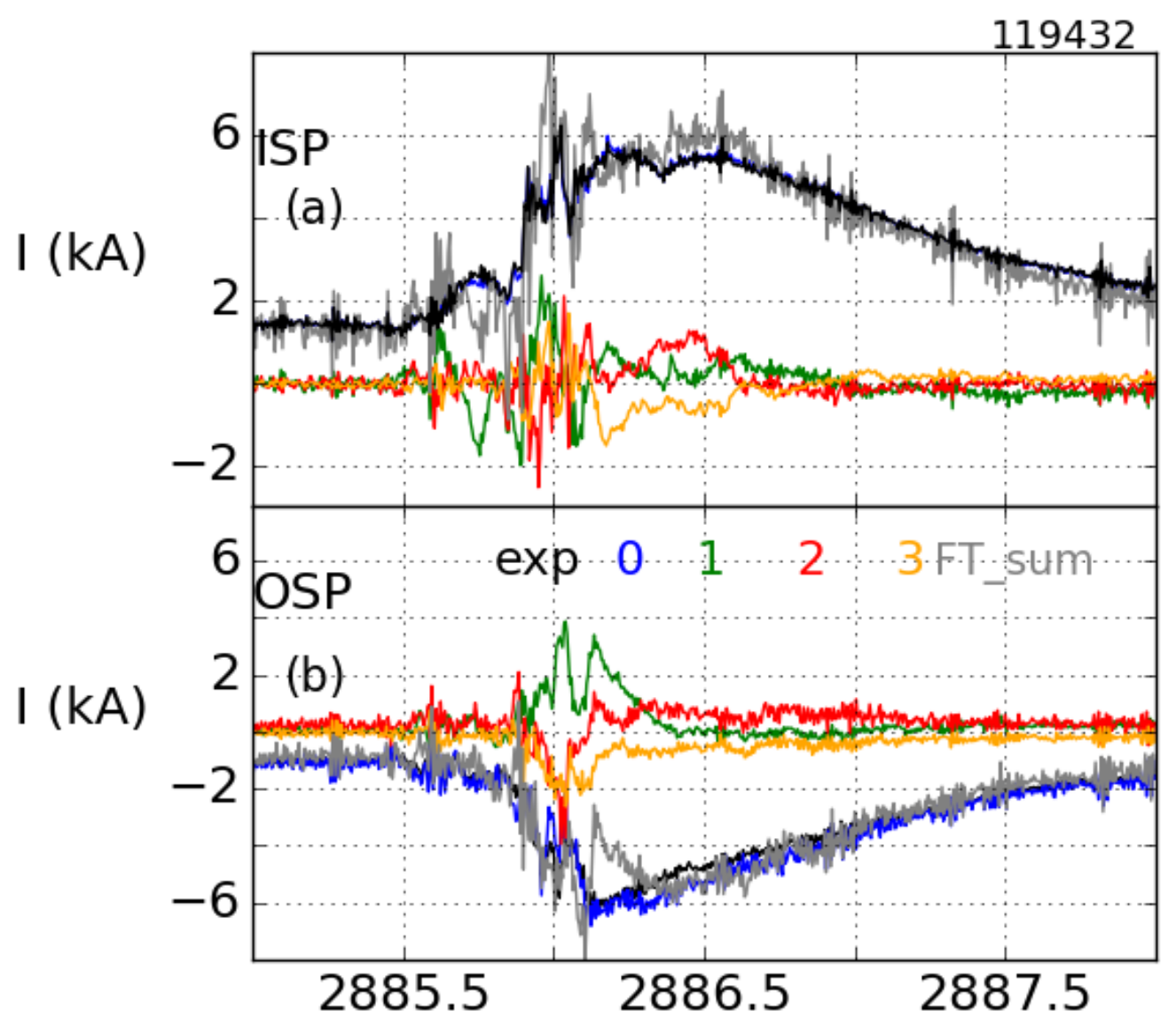

Figure 9 Mode decomposition of tile currents during type-I ELM: a) ISP Amplitude of $n=0$ to $n=3$ modes b) OSP Amplitude of $n=0$ to $n=3$ modes during a typical ELM. The sum of the currents in the decomposed modes (grey) is compared to the experimental measurement (black), the difference is due to missing higher harmonics in the decomposition and finite tile width effects (radial broadening of divertor current deposition during the ELM, currents in private flux region and outer SOL not captured by central strike point tile). 


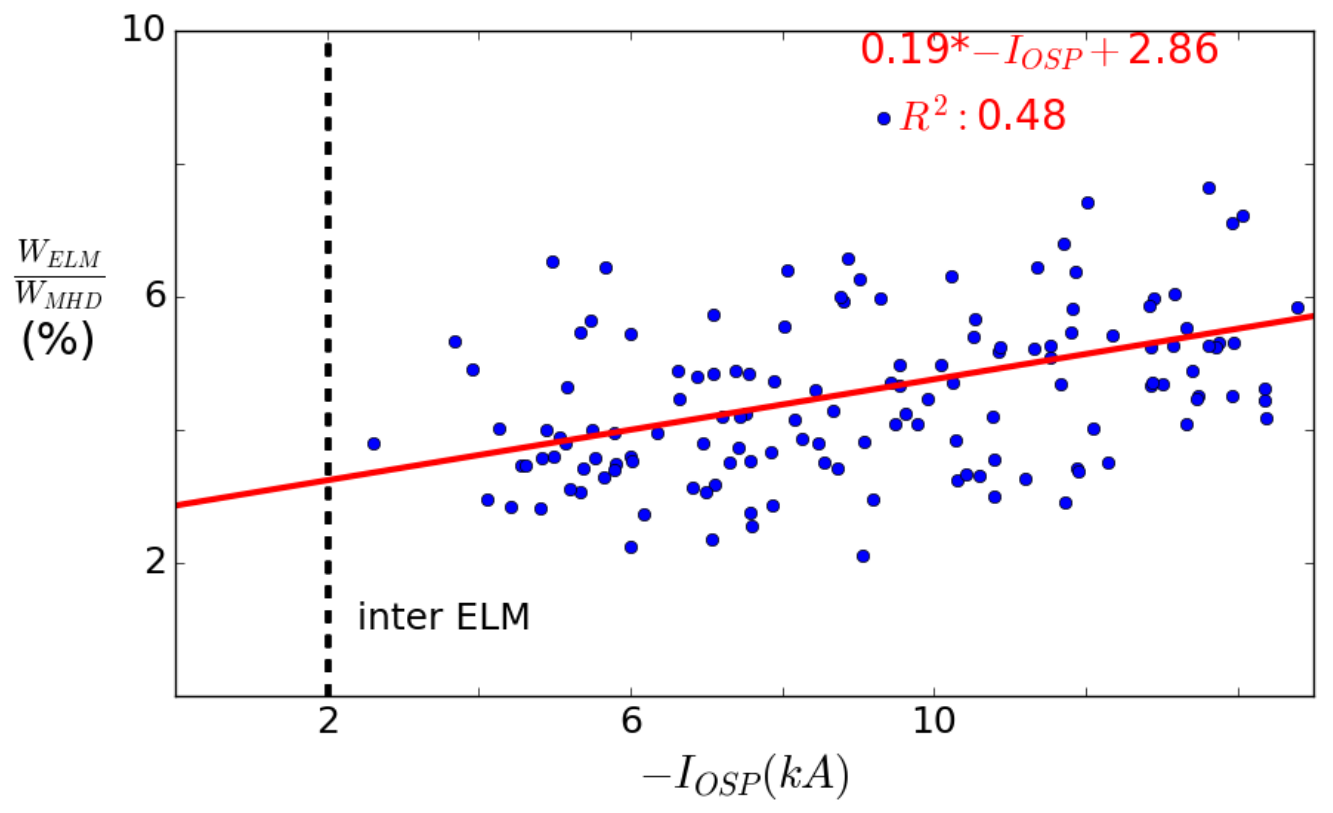

Figure 10 Dependence of ELM size as percentage of the total stored energy on currents near the OSP in A on discharges 121552-121571. The x-axis is the peak amplitude of the total current $I_{n=0}$ from Eq. (2) in the oscillatory phase. 


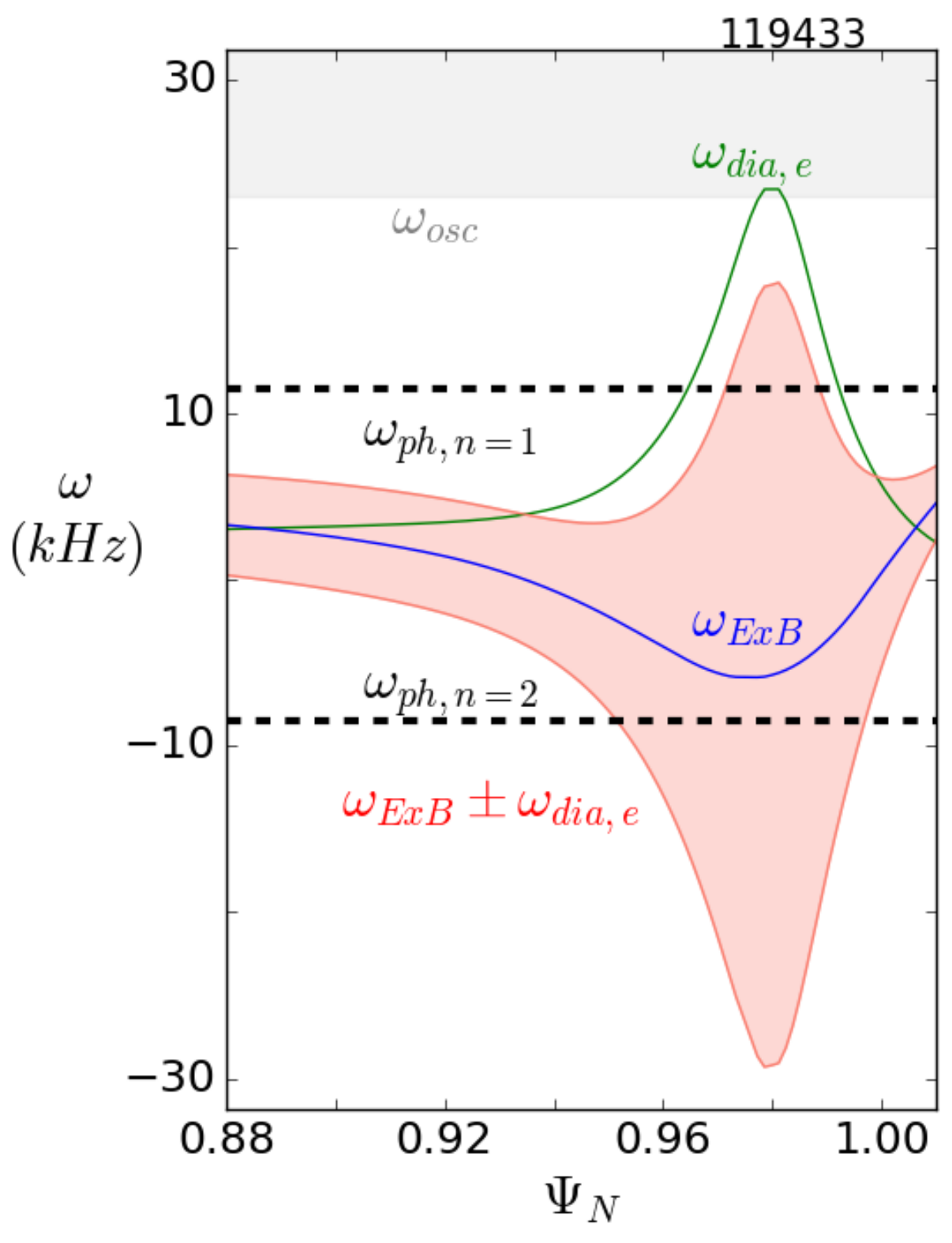

Figure 11 Diamagnetic (green) and ExB rotation (blue) frequency at the plasma edge (based on conditional averaging of $80-99 \%$ of the ELM phase), range of expected rotation from simulations [29] (red) and TCA (black, dashed lines) during discharge 119433. 


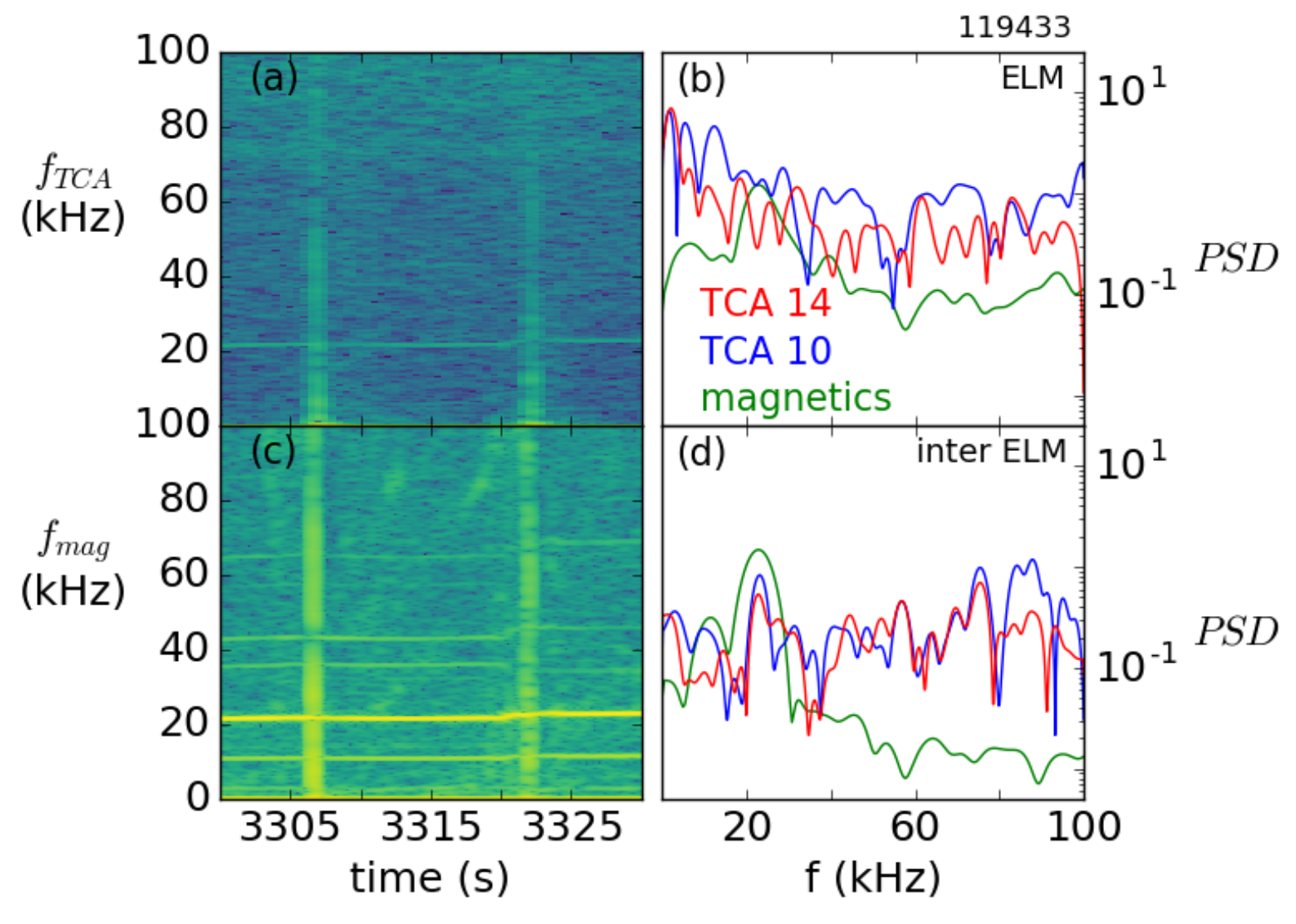

Figure 12 Power spectral density in TCA and magnetics during discharge 119433 (left), spectrum at selected times (right) during an ELM (3321.85 $\pm 0.25 \mathrm{~ms})$ and in the inter ELM phase (3326.05 $\pm 0.25 \mathrm{~ms}$ ) for a fast magnetic probe (green), a TCA sensor near the inner (blue) and outer (red) strike point. The power is plotted as square root of the power spectral density. 


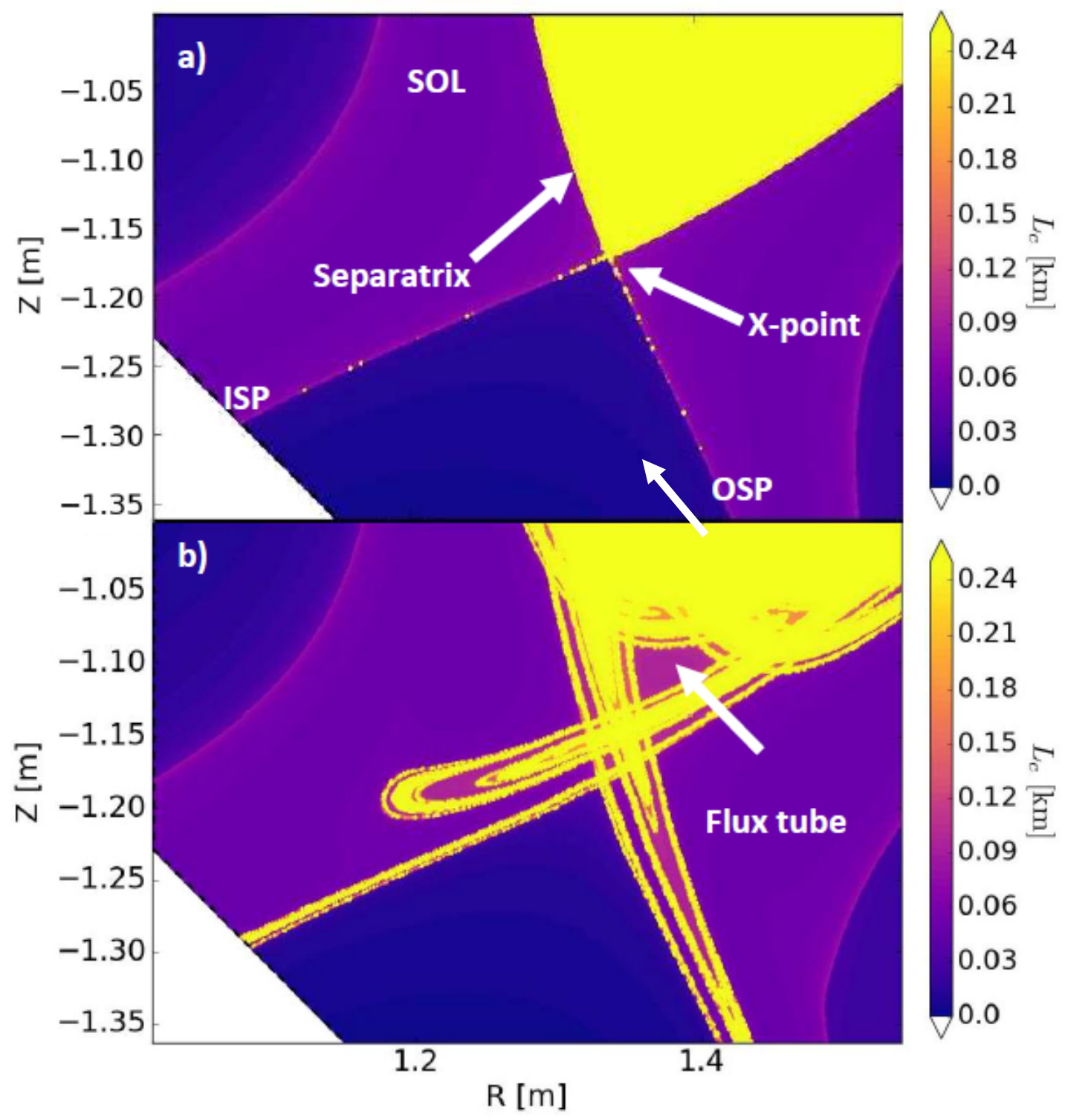

Figure 13 MAFOT connection length calculation of discharge 121560, based on a) solely kinetic equilibrium b) additional error fields from C-Coil, F-Coil and B-coil: Spatial location of short connection length flux tubes between the inner and outer divertor inside of the separatrix and their respective length $L_{c}$. 


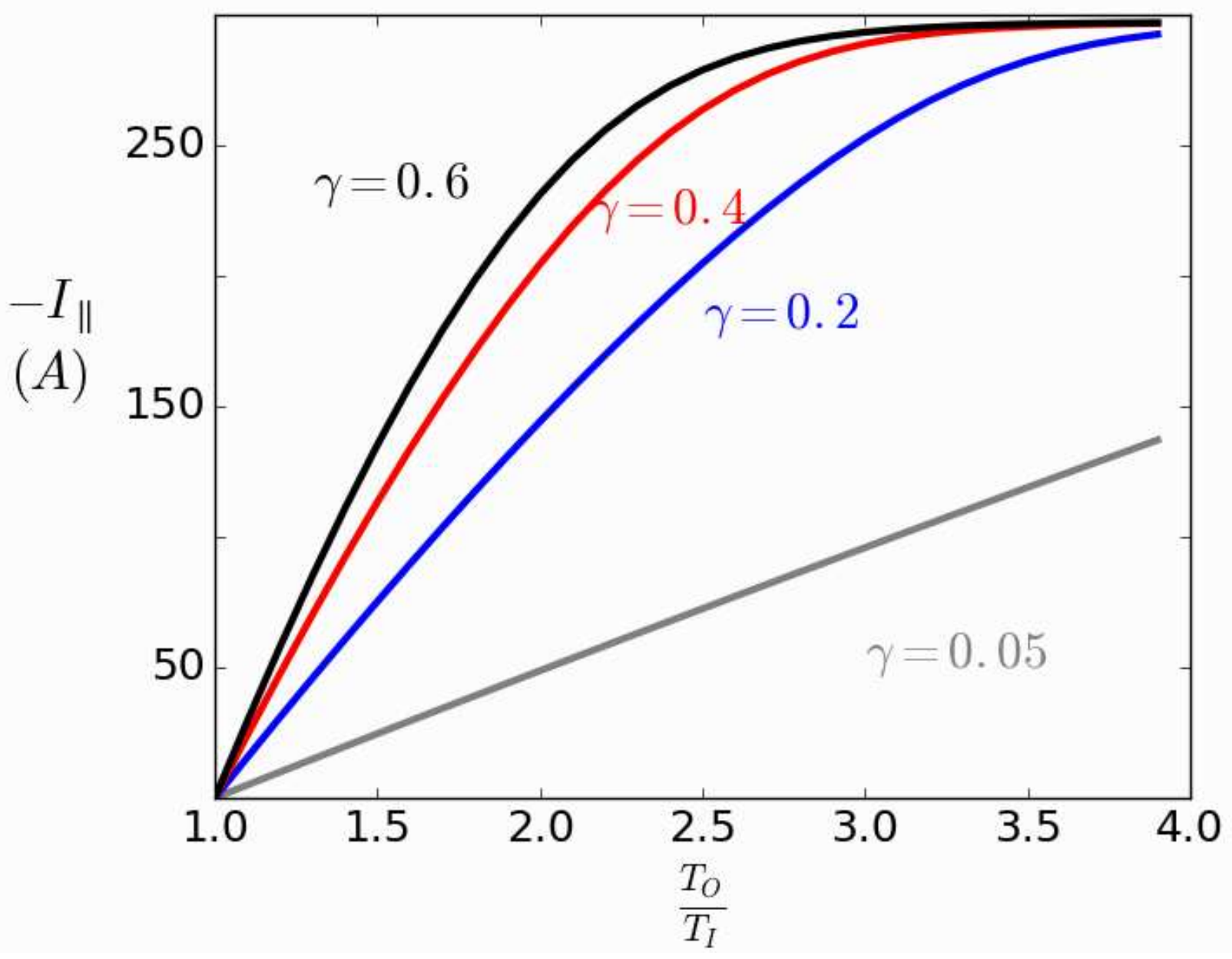

Figure 14 Calculated size of the thermoelectric currents at the end of a $100 \mathrm{~m}$ long connecting flux tubes versus the temperature ratio at the end of the tubes for different values of the conductance-like parameter $\gamma$. The experimental value is $\gamma=0.35$. 


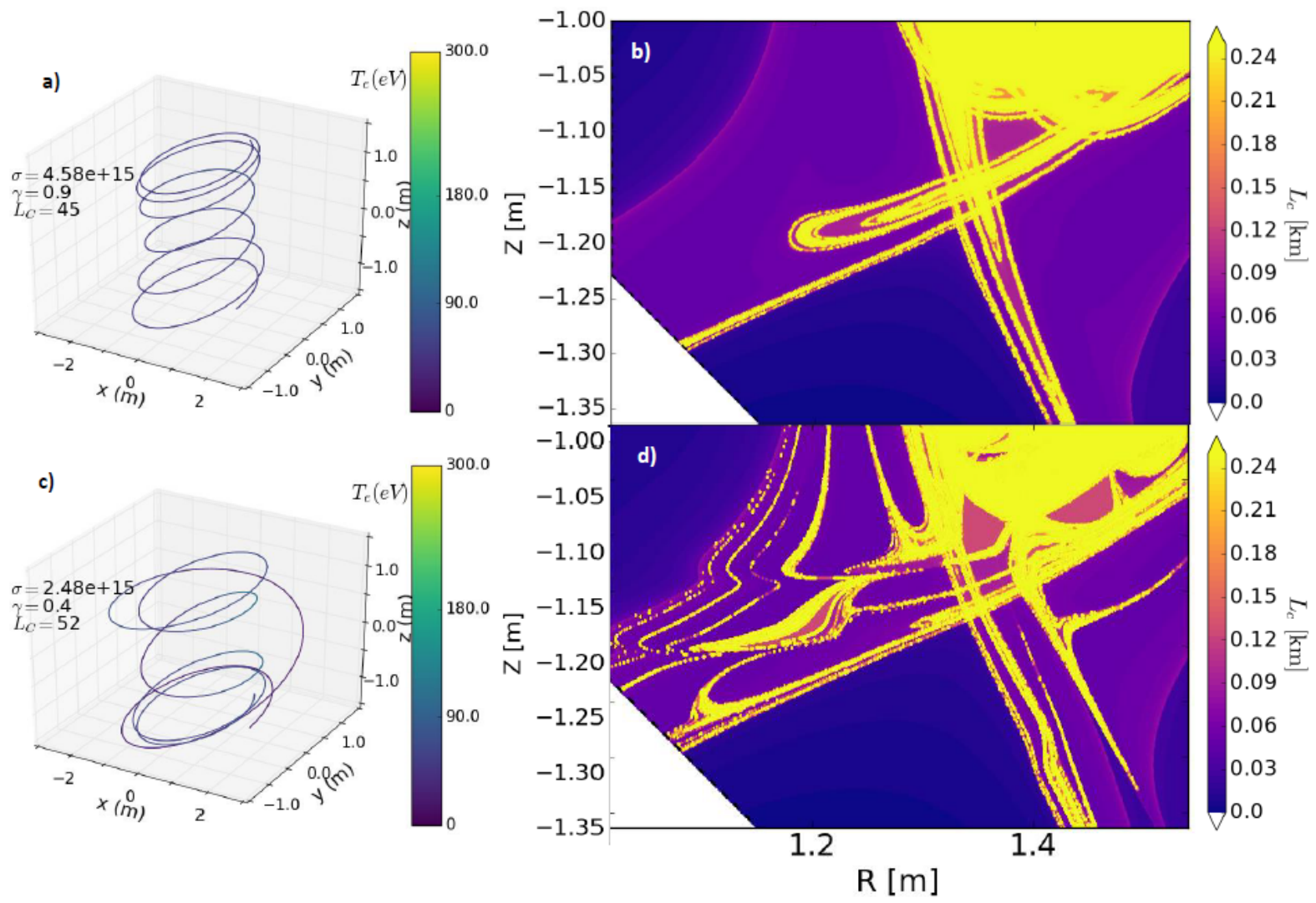

Figure 15 MAFOT simulation of discharge 121560 as shown in Figure 13 now with an additional current flow of $500 \mathrm{~A}$ along an SOL field line (a) with the connection length $\mathrm{L}_{c}$ calculation (b) or along a flux tube through the plasma (c) with its connection length $L_{c}$ calculation (d) 

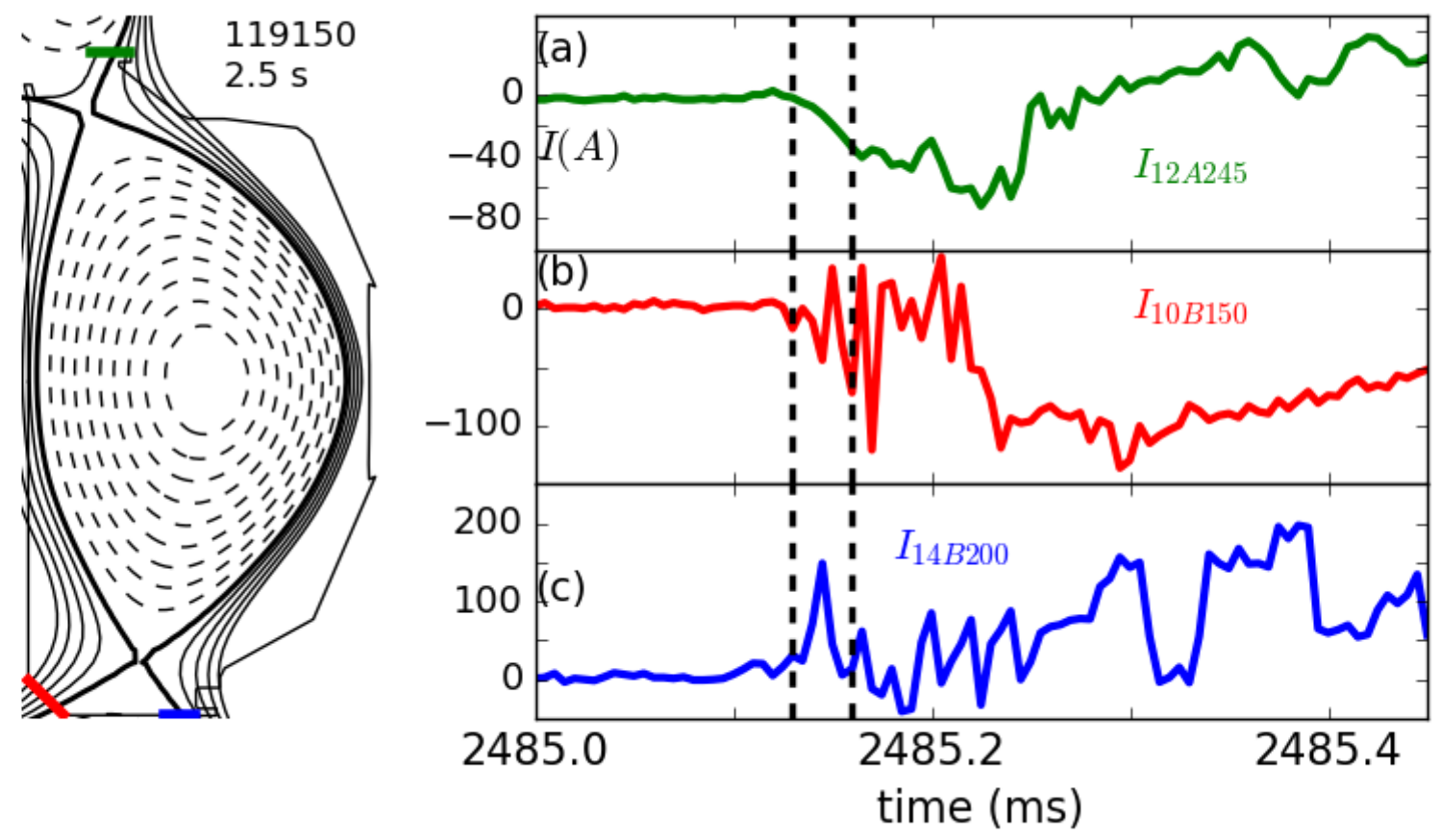

Figure 16 Tile current evolution during $1 \mathrm{~ms}$ ELM in DN discharge 119150 (EFIT, left): TCA measurements from upper OSP (a), lower ISP (b) and lower OSP (c). The dashed lines mark the 0.03 ms time interval needed for the ELM to spread from LFS to HFS. 

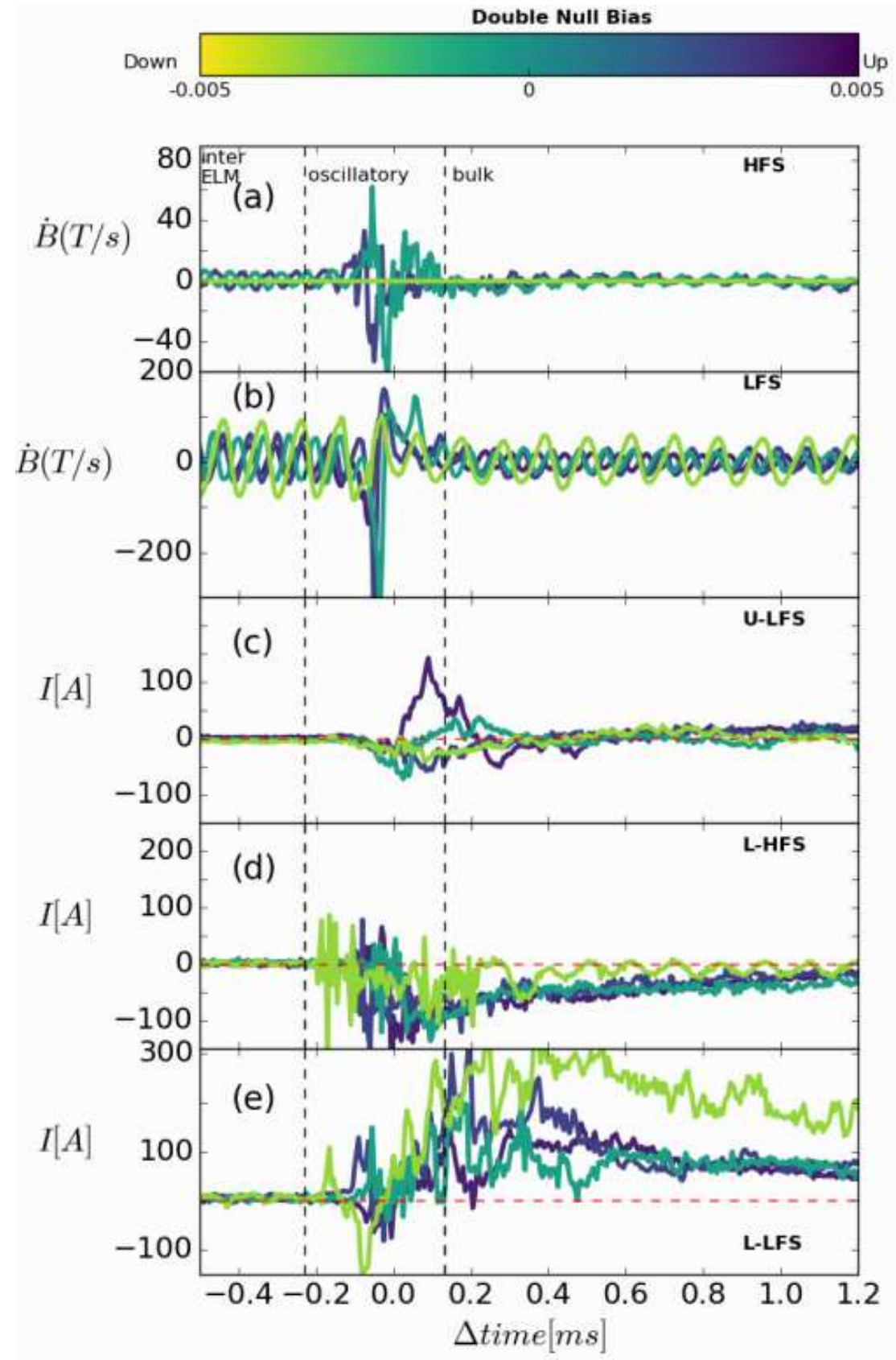

Figure 17 ELM evolution in dependence of magnetic balance on discharge 119150: Magnetic probe signals relative to Da peak (not shown) on a) HFS (322 degree, no data available for biased down case) b) LFS (167 degree); Tile current measurements on c) upper low field side, d) lower high field side and e) lower low field side. Typical ELMs for upwards bias (blue), downwards bias (lime green) and balanced DN shapes (turquoise). 0.3 ms oscillatory time window for comparison to purely LSN ELMs. 


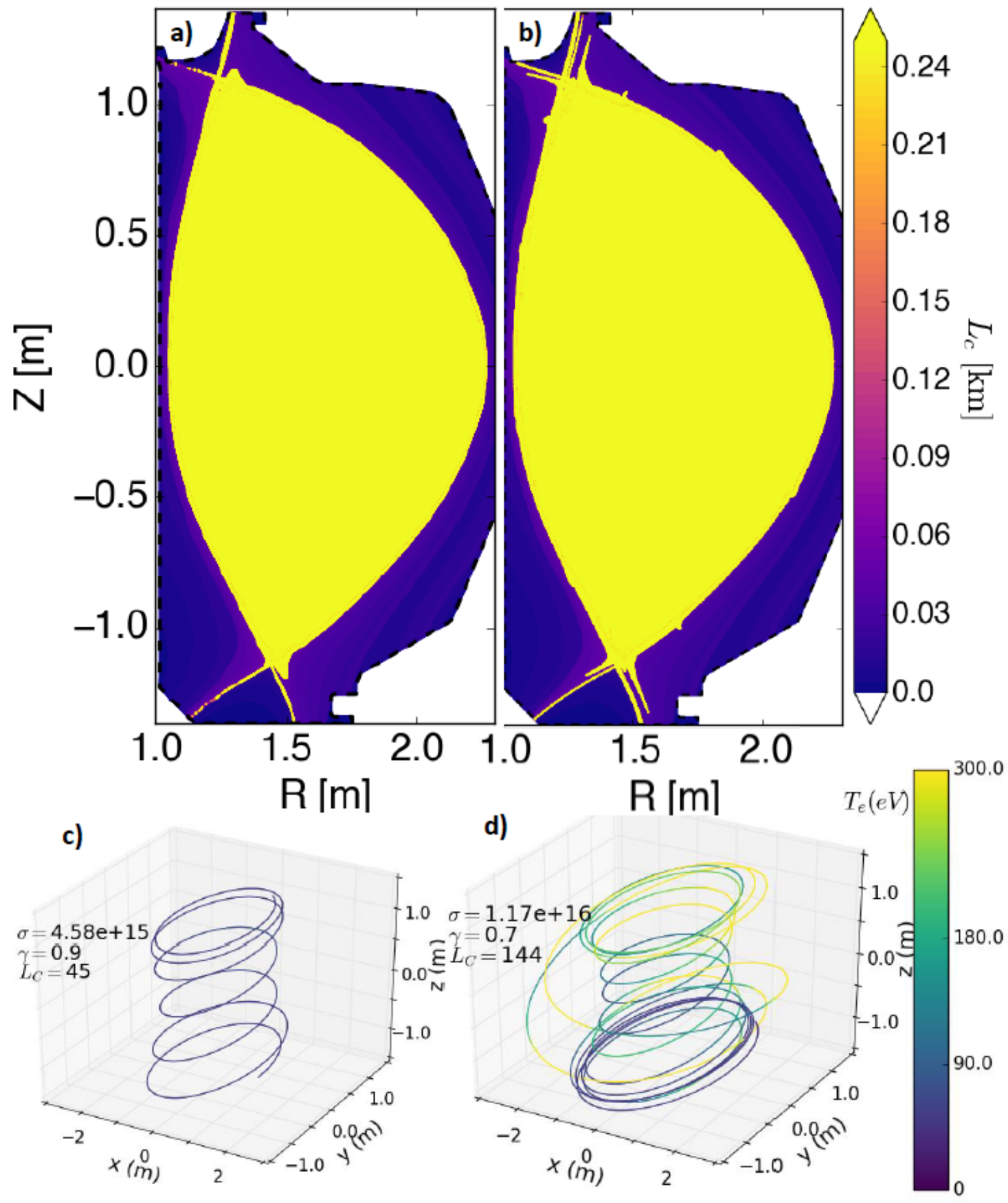

Figure 18 MAFOT connection length Lc calculation of discharge 119150 with a) error fields only b) one filament with $150 \mathrm{~A}$ on the LFS A: c) representative field line in the HFS near strike point SOL d) flux tube through the plasma connecting LFS and HFS 
*This material is based upon work supported by the U.S. Department of Energy, Office of Science, Office of Fusion Energy Sciences, under Award Numbers DE-FG02-07ER54917, DE-SC0018030 and DE-FG02-05ER54809. This research used resources of the DIII-D National Fusion Facility, which is a DOE Office of Science User Facility. We gratefully acknowledge the support of the DIII-D Team for tokamak, auxiliary heating, and diagnostic systems operation. Disclaimer: This report was prepared as an account of work sponsored by an agency of the United States Government. Neither the United States Government nor any agency thereof, nor any of their employees, makes any warranty, express or implied, or assumes any legal liability or responsibility for the accuracy, completeness, or usefulness of any information, apparatus, product, or process disclosed, or represents that its use would not infringe privately owned rights. Reference herein to any specific commercial product, process, or service by trade name, trademark, manufacturer, or otherwise does not necessarily constitute or imply its endorsement, recommendation, or favoring by the United States Government or any agency thereof. The views and opinions of authors expressed herein do not necessarily state or reflect those of the United States Government or any agency thereof. 


\section{REFERENCES}

[1] Hawryluk R J and et al. 2009 Principal physics developments evaluated in the ITER design review Nucl. Fusion 49065012

[2] Leonard A W 2014 Edge-localized-modes in tokamaksa) Phys. Plasmas 21090501

[3] Loarte A, Saibene G, Sartori R, Campbell D, Becoulet M, Horten L, Eich T, Herrmann A, Matthews G, Asakura N, Chankin A, Leonard A, Porter G, Federici G, Janeschitz G, Shimada M and Sugihara M 2003 Characteristics of type I ELM energy and particle losses in existing devices and their extrapolation to ITER Plasma Phys. Control. Fusion 45 1549-69

[4] Snyder P B, Wilson H R, Ferron J R, Lao L L, Leonard A W, Osborne T H, Turnbull A D, Mossessian D, Murakami M and Xu X Q 2002 Edge localized modes and the pedestal: A model based on coupled peeling-ballooning modes Physics of Plasmas vol 9 pp 2037-43

[5] Eich T, Sieglin B, Thornton A J, Faitsch M, Kirk A, Herrmann A and Suttrop W 2017 ELM divertor peak energy fluence scaling to ITER with data from JET, MAST and ASDEX upgrade Nucl. Mater. Energy 12 84-90

[6] Sugiyama L E and Strauss H R 2010 Magnetic X-points, edge localized modes, and stochasticity Phys. Plasmas 17

[7] Pamela S J P, Huijsmans G T A, Eich T, Saarelma S, Lupelli I, Maggi C F, Giroud C, Chapman I T, Smith S F, Frassinetti L, Becoulet M, Hoelzl M, Orain F and Futatani S 2017 Recent progress in the quantitative validation of JOREK simulations of ELMs in JET Nucl. Fusion $\mathbf{5 7}$

[8] Pamela S, Eich T, Frassinetti L, Sieglin B, Saarelma S, Huijsmans G, Hoelzl M, Becoulet M, Orain F, Devaux S, Chapman I, Lupelli I, Solano E and Contributors J E T 2015 Non-linear MHD simulations of ELMs in JET and quantitative comparisons to experiments Plasma Phys. Control. Fusion $\mathbf{5 8}$

[9] Evans T E, Yu J H, Jakubowski M W, Schmitz O, Watkins J G and Moyer R A 2009 A conceptual model of the magnetic topology and nonlinear dynamics of ELMs J. Nucl. Mater. 390-391 789-92

[10] Wingen A, Evans T E, Lasnier C J and Spatschek K H 2010 Numerical modeling of edge-localizedmode filaments on divertor plates based on thermoelectric currents Phys. Rev. Lett. 104

[11] Harbour P J, Summers D D R, Clement S, Coad J P, De Kock L, Ehrenberg J, Erents K, Gottardi N, Hubbard A, Keilhacker M, Morgan P D, Snipes J A, Stamp M F, Tagle J A, Tanga A, Behrisch R and Wang W M 1989 The X-point scrape-off plasma in jet with L- and H-modes J. Nucl. Mater. 162164 236-44

[12] Pitts R ., Alberti S, Blanchard P, Horacek J, Reimerdes H and Stangeby P . 2003 ELM driven divertor target currents on TCV Nucl. Fusion 43 1145-66

[13] Kallenbach A, Carlson A, Pautasso G, Peeters A, Seidel U and Zehrfeld H P 2001 Electric currents in the scrape-off layer in ASDEX Upgrade J. Nucl. Mater. 290-293 639-43

[14] Schaffer M J and Leikind B J 1991 Observation of electric currents in diverted tokamak scrape-off layers Nucl. Fusion 31 1750-8 
[15] Evans T E, Lasnier C J, Hill D N, Leonard A W, Fenstermacher M E, Petrie T W and Schaffer M J 1995 Measurements of non-axisymmetric effects in the DIII-D divertor J. Nucl. Mater. 220-222 235-9

[16] Knolker M, Bortolon A, Canal G P, Evans T E, Zohm H, Abrams T, Buttery R J, Davis E M, Groebner R J, Hollmann E, Fenstermacher M E, Lasnier C, Leonard A W, Moyer R A, Nazikian R, Osborne T $\mathrm{H}, \mathrm{Paz}-$ Soldan C and Sieglin B 2018 Investigation of the role of pedestal pressure and collisionality on type-I ELM divertor heat loads in DIII-D Nucl. Fusion 58096023

[17] Fenstermacher M E, Leonard A W, Snyder P B, Boedo J A, Brooks N H, Colchin R J, Gray D S, Groebner R J, Groth M, Hollmann E M, Lasnier C J, Osborne T H, Petrie T W, Rudakov D L, Takahashi H, Watkins J G and Zeng L 2003 ELM particle and energy transport in the SOL and divertor of DIII-D Plasma Phys. Control. Fusion 45 1597-626

[18] Martin Y R and Takizuka T 2008 Power requirement for accessing the H-mode in ITER J. Phys. Conf. Ser. 123

[19] Leonard A W, Casper T A, Groebner R J, Osborne T H, Snyder P B and Thomas D M 2007 Pedestal performance dependence upon plasma shape in DIII-D Nuclear Fusion vol 47 pp 552-62

[20] Solomon W M, Burrell K H, Garofalo A M, Groebner R J, Lasnier C J, Makowski M A, Osborne TH, Reimerdes H, Degrassie J S, Doyle E J, Evans T E, Fenstermacher M E, Jackson G L and Schaffer M J 2012 ELM pacing using modulated non-axisymmetric magnetic fields on DIII-D Nucl. Fusion $\mathbf{5 2}$

[21] Herrmann A, Junker W, Gunther K, Bosch S, Kaufmann M, Neuhauser J, Pautasso G, Richter T and Schneider R 1995 Energy flux to the ASDEX-Upgrade diverter plates determined by thermography and calorimetry Plasma Phys. Control. Fusion 37 17-29

[22] Snyder P B, Wilson H R and Xu X Q 2005 Progress in the peeling-ballooning model of edge localized modes: Numerical studies of nonlinear dynamics Physics of Plasmas vol $12 \mathrm{pp} 1-7$

[23] Kirk A, Dunai D, Dunne M, Huijsmans G, Pamela S, Becoulet M, Harrison J R, Hillesheim J, Roach C and Saarelma S 2014 Recent progress in understanding the processes underlying the triggering of and energy loss associated with type i ELMs Nucl. Fusion $\mathbf{5 4}$

[24] Takahashi H, Fredrickson E D, Schaffer M J, Austin M E, Evans T E, Lao L L and Watkins J G 2004 Observation of SOL current correlated with MHD activity in NBI heated DIII-D tokamak discharges Nucl. Fusion 44 1075-96

[25] Takahashi H, Fredrickson E D and Schaffer M J 2008 Scrape-off-layer current model for filament structure observed during edge-localized modes in the DIII-D tokamak Phys. Rev. Lett. 100

[26] Krebs I, Hölzl M, Lackner K and Günter S 2013 Nonlinear excitation of low-n harmonics in reduced magnetohydrodynamic simulations of edge-localized modes Phys. Plasmas $\mathbf{2 0} 082506$

[27] Xia T Y and Xu X Q 2015 Nonlinear fluid simulation of particle and heat fluxes during burst of ELMs on DIII-D with BOUT++ code Nucl. Fusion

[28] Wade M R, Burrell K H, Leonard A W, Osborne T H and Snyder P B 2005 Edge-localized-modeinduced transport of impurity density, energy, and momentum Phys. Rev. Lett. 
[29] UZAWA K, ISHIZAWA A and NAKAJIMA N 2010 Intrinsic Rotation of a Magnetic Island with Finite Width Plasma Fusion Res. 5 S1016-S1016

[30] Wenninger R P, Zohm H, Boom J E, Burckhart A, Dunne M G, Dux R, Eich T, Fischer R, Fuchs C, Garcia-Munoz M, Igochine V, Hölzl M, N.c. L, Lunt T, Maraschek M, Müller H W, Park H K, Schneider P A, Sommer F, Suttrop W and Viezzer E 2012 Solitary magnetic perturbations at the ELM onset Nucl. Fusion $\mathbf{5 2}$

[31] Spolaore M, Kovařík K, Stöckel J, Adamek J, Dejarnac R, Ďuran I, Komm M, Markovic T, Martines E, Panek R, Seidl J and Vianello N 2017 Electromagnetic ELM and inter-ELM filaments detected in the COMPASS Scrape-Off Layer Nucl. Mater. Energy 12 844-51

[32] Wingen A, Evans T E and Spatschek K H 2009 High resolution numerical studies of separatrix splitting due to non-axisymmetric perturbation in DIII-D Nucl. Fusion 49

[33] Evans T E, Moyer R A and Monat P 2002 Modeling of stochastic magnetic flux loss from the edge of a poloidally diverted tokamak Phys. Plasmas 9 4957-67

[34] Roeder R K W, Rapoport B I and Evans T E 2003 Explicit calculations of homoclinic tangles in tokamaks Phys. Plasmas

[35] Evans T E, Roeder R K W, Carter J A and Rapoport B I 2004 Homoclinic tangles, bifurcations and edge stochasticity in diverted tokamaks Contributions to Plasma Physics

[36] Evans T E, Roeder R K W, Carter J A, Rapoport B I, Fenstermacher M E and Lasnier C J 2005 Experimental signatures of homoclinic tangles in poloidally diverted tokamaks J. Phys. Conf. Ser.

[37] Huijsmans G T A and Loarte A 2013 Non-linear MHD simulation of ELM energy deposition Nucl. Fusion 53

[38] Wingen A, Evans T E and Spatschek K H 2011 Effect of thermoelectric current splitting on the magnetic topology in DIII-D Phys. Plasmas 18

[39] Moyer R A, Bykov I, Orlov D M, Evans T E, Lee J S, Teklu A M, Fenstermacher M E, Makowski M, Lasnier C J, Wang H Q, Watkins J G and Wu W 2018 Imaging divertor strike point splitting in RMP ELM suppression experiments in the DIII-D tokamak Rev. Sci. Instrum. 89 10E106

[40] Kirk A, Koch B, Scannell R, Wilson H R, Counsell G, Dowling J, Herrmann A, Martin R and Walsh M 2006 Evolution of filament structures during edge-localized modes in the MAST tokamak Phys. Rev. Lett.

[41] Rack M, Wingen A, Liang Y, Spatschek K H, Harting D M and Devaux S 2012 Thermoelectric currents and their role during ELM formation in JET Nucl. Fusion $\mathbf{5 2}$

[42] Asdex Upgrade Team T, Faitsch M, Sieglin B, Eich T, Herrmann A and Suttrop W 2017 Divertor heat load in ASDEX Upgrade L-mode in presence of external magnetic perturbation Plasma Phys. Control. Fusion 59

[43] Staebler G M and Hinton F L 1989 Currents in the scrape-off layer of diverted tokamaks Nucl. Fusion 29 1820-4

[44] Pitts R A, Andrew P, Arnoux G, Eich T, Fundamenski W, Huber A, Silva C and Tskhakaya D 2007 
ELM transport in the JET scrape-off layer Nucl. Fusion 47 1437-48

[45] Staebler G M 1995 Divertor bias experiments J. Nucl. Mater.

[46] Wingen A, Ferraro N M, Shafer M W, Unterberg E A, Evans T E, Hillis D L and Snyder P B 2014 Impact of plasma response on plasma displacements in DIII-D during application of external 3D perturbations Nucl. Fusion

[47] Stangeby P C, Elder J D, McLean A G and Watkins J G 2017 Experimentally-based ExB drifts in the DIII-D divertor and SOL calculated from integration of Ohm's law using Thomson scattering measurements of Te and ne Nucl. Mater. Energy 12 876-81

[48] Staebler G M 1996 The critical point for the onset of divertor energy flux asymmetry in tokamaks Nucl. Fusion 36 1437-53

[49] Petrie T W, Watkins J G, Lao L L and Snyder P B 2003 The role of magnetic geometry on the poloidal distribution of ELM-induced peak particle flux at the divertor targets in DIII-D Nucl. Fusion

[50] Nunes I, Conway G D, Loarte A, Manso M, Serra F and Suttrop W 2004 Characterization of the density profile collapse of type I ELMs in ASDEX Upgrade with high temporal and spatial resolution reflectometry Nucl. Fusion

[51] Kallenbach A, Dux R, Eich T, Fischer R, Giannone L, Harhausen J, Herrmann A, Müller H W, Pautasso $G$ and Wischmeier M 2008 Divertor power and particle fluxes between and during typeI ELMs in the ASDEX Upgrade Nuclear Fusion vol 48

[52] Eich T, Kallenbach A, Pitts R A, Jachmich S, Fuchs J C, Herrmann A and Neuhauser J 2007 Divertor power deposition and target current asymmetries during type-I ELMs in ASDEX Upgrade and JET J. Nucl. Mater. 363-365 989-93

[53] Joseph I, Cohen R H and Ryutov D D 2009 Driving toroidally asymmetric current through the tokamak scrape-off layer. I. Potential for edge localized mode suppression Physics of Plasmas vol 16

[54] Schaffer M J, Mahdavi A, Klepper C C, Hill D N and Rensink M E 1992 Effect of divertor bias on plasma flow in the DIII-D scrape-off layer Nucl. Fusion 32 855-61

[55] Zweben S J, Maqueda R J, Roquemore A L, Bush C E, Kaita R, Marsala R J, Raitses Y, Cohen R H and Ryutov D D 2009 Biased electrodes for SOL control in NSTX J. Nucl. Mater. 390-391 417-20

[56] Zweben S J, Campanell M D, Lyons B C, Maqueda R J, Raitses Y, Roquemore A L, Scotti F and Takahashi H 2012 Local effects of biased electrodes in the divertor of NSTX Plasma Phys. Control. Fusion 54 
